\title{
Equilibrium properties of disordered spin models with two-scale interactions
}

\author{
Jack Raymond ${ }^{1, *}$ and David Saad ${ }^{2, \dagger}$ \\ ${ }^{1}$ Hong Kong University of Science and Technology, Clear Water Bay Road, Clear Water Bay, Hong Kong \\ ${ }^{2}$ Neural Computing Research Group, Aston University, Birmingham, United Kingdom
}

(Received 1 June 2009; published 24 September 2009)

\begin{abstract}
Methods for understanding classical disordered spin systems with interactions conforming to some idealized graphical structure are well developed. The equilibrium properties of the Sherrington-Kirkpatrick model, which has a densely connected structure, have become well understood. Many features generalize to sparse ErdösRényi graph structures above the percolation threshold and to Bethe lattices when appropriate boundary conditions apply. In this paper, we consider spin states subject to a combination of sparse strong interactions with weak dense interactions, which we term a composite model. The equilibrium properties are examined through the replica method, with exact analysis of the high-temperature paramagnetic, spin-glass, and ferromagnetic phases by perturbative schemes. We present results of replica symmetric variational approximations, where perturbative approaches fail at lower temperature. Results demonstrate re-entrant behaviors from spin glass to ferromagnetic phases as temperature is lowered, including transitions from replica symmetry broken to replica symmetric phases. The nature of high-temperature transitions is found to be sensitive to the connectivity profile in the sparse subgraph, with regular connectivity a discontinuous transition from the paramagnetic to ferromagnetic phases is apparent.
\end{abstract}

DOI: 10.1103/PhysRevE.80.031138

PACS number(s): 75.10.Hk, 05.20.-y, 75.10.Nr, 75.50.Lk

\section{INTRODUCTION}

Statistical physics methods for studying disordered spin systems have become well developed. Much of the development can be traced back to early work on mean-field models for disordered magnetic systems and the theory was strongly developed in spin-glass models $[1,2]$. One problem in studying spin glasses and disordered media has been in appropriately modeling the inhomogeneity within tractable frameworks. Statistical descriptions of inhomogeneity are often realized by random coupling ensembles. Small systems described in this way may have strongly varying properties, but the ensemble may be chosen so that the macroscopic description is asymptotically well defined.

Both dense and sparse graphical models are useful in understanding a range of phenomena, such as neural networks [3], information theory [4], and other information processing [5], where spatial and dimensional constraints are often less rigid. Many complex systems have an inhomogeneous interaction structure that can be approached, if not exactly represented, by consideration of simple random graph ensembles. In this paper, spin-glass models with couplings conforming to infinite-dimensional Erdös-Rényi random graphs are considered [6]. In the large system limit, many equilibrium properties depend on the connectivity distribution and how the number of couplings per variable scales with $N$, the system size. Dense graphs have a number of links per variable that is $O(N)$ in the large system limit, whereas sparse ensembles have finite mean connectivity in this limit. Many topological features become well defined in these limits. Two standard sparse coupling distributions are considered: a description with regular connectivity, and one with Poissonian connec-

\footnotetext{
*jack.raymond@physics.org; http://ihome.ust.hk/ jraymond

†saadd@aston.ac.uk
}

tivity. The distinctions between these two sparse models and the limiting case of full connectivity are illustrated in Fig. 1.

Some densely connected models may be analyzed exactly for ensembles of uniform binary interactions, and certain random coupling models, most famously the SherringtonKirkpatrick (SK) model of spin glasses [7]. Simplification of the analysis in the disordered case is often possible through noting the ability to describe large sets of interactions by central limit theorems [8]. For sparse connectivity models, such as the Viana-Bray (VB) model $[9,10]$, a locally treelike approximation (Bethe approximation) is often essential in simplifying analysis; central limit theorems again apply to certain objects but not directly to the set of local interactions for any variable. Models, which do not allow the use of central limit theorems or locally treelike approximations, are normally significantly more difficult to analyze.

Frameworks in which an interplay between strong sparse and weak pervasive couplings might be proposed in a variety of areas. In nanotechnology, for example, miniaturization of classical components will preserve engineered short-range interactions, but other accidental correlations may emerge not limited by the designed connectivity structure, and these may well be modeled by a mean-field (infinite connectivity) like interaction. A mixed connectivity may also be a designed feature. Neuronal activity is known to involve a combination of short- and long-range information processing structures, this motivated a $1+\infty$-dimensional model of neuronal activity [11] discovering many novel properties. Another example of such an engineering application is in the bandwidth allocation in multiuser communication systems, where improvement over standard methods is possible [12].

To motivate a closely related study, Hase and Mendes noted a possible application for theories of these structures [13]. Consider the model with sparse antiferromagnetic (AF) couplings on a structure, otherwise, fully connected through ferromagnetic couplings. This composite model can be considered as one in which a ferromagnetic phase is maintained 

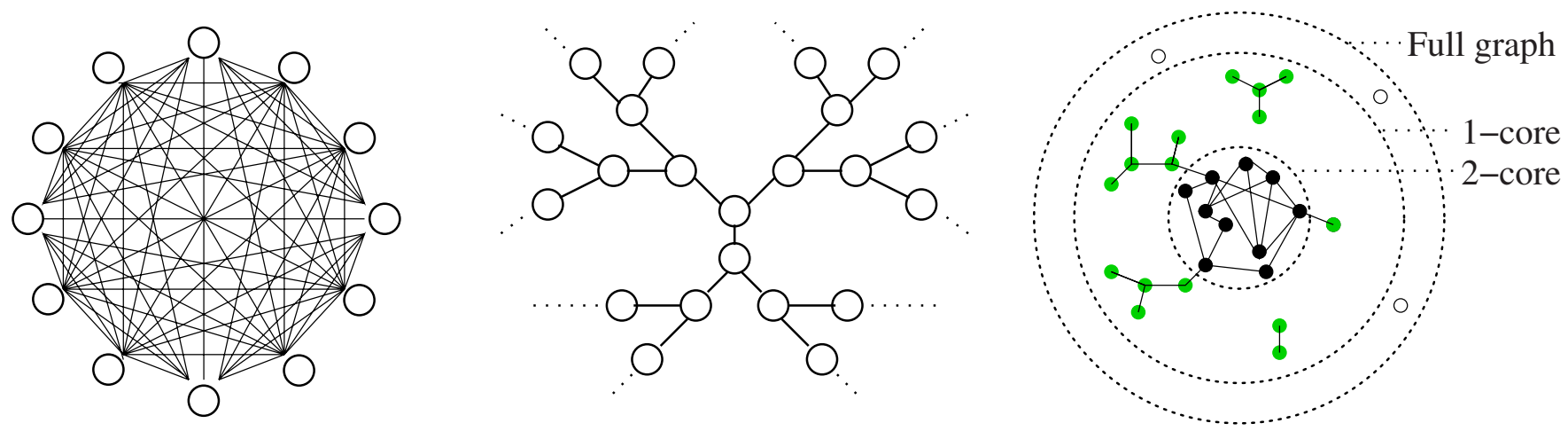

FIG. 1. (Color online) Shown are the couplings (links) among a set of spin variables (circles), which describes graphically a particular quadratic Hamiltonian. Left figure: the fully connected graph is a special case of a dense graph describing the SK model, with $O(N)$ nonzero couplings per variable in the large system limit. Centre/right figure: the VB model is defined with $O(1)$ nonzero couplings per variable in the large system limit. Center figure: in the case of a regular connectivity random graph above the percolation threshold, there is an inhomogeneous structure on a global scale, but locally the structure is a Bethe lattice (regular tree). Right figure: in the case of a random graph with Poissonian connectivity the local structure is again treelike. Above the percolation threshold many trees of finite size and unconnected variables exist, as well as a giant component containing $O(N)$ variables, and many loops [6]. The 1 core contains all variables with at least one link, including the giant component above the percolation threshold. Additional structures within the giant component may be identified, including a 2 core, obtained by recursively removing leaves (singly connected variables) from the giant component.

by a densely connected network, but with a small proportion of links attacked. Often only a small portion of a link structure is accessible to an attacker, so it is interesting to consider how the system response differs from weak attacks on all (or most) links.

The effect of an attack on a sparse subset may cause a transition away from the ordered phase, when sufficiently strong. It is possible that the nature of transitions away from the ordered state may differ from those with only a single interaction scale. The effect of disruption of networks by random attack, or frustrating interactions, is of importance in many practical network models $[13,14]$, the restriction to random topologies allows a focus on generic properties, in this case restricted to the issue of sparse- and dense-induced effects.

More generally, a range of mean-field behavior, including spin glasslike, may be supported by the dense substructure combined with an arbitrary set supported by the sparse sub- structure, as shown in Fig. 2. In so doing, a wider variety of competitive phase behavior is explored.

It may be expected that many of the results for composite systems will be similar to those for the limiting sparse and dense models. Four thermodynamic phases describe equilibrium properties of spin models with independent and identically distributed (iid) couplings: a pure state with no macroscopic order, the paramagnetic phase; a pure state with macroscopic order aligned with some mean bias in the couplings, the replica symmetric (RS) ferromagnetic (F) phase; a macroscopically aligned phase, but with some complicated phase-space fragmentation, the mixed (M) phase; and a phase with no macroscopic alignment and a complicated fragmentation of the phase space, the spin-glass phase (SG). Within both the sparse and dense Ising spin models, these phases are exhibited and many features are shared by the two models.
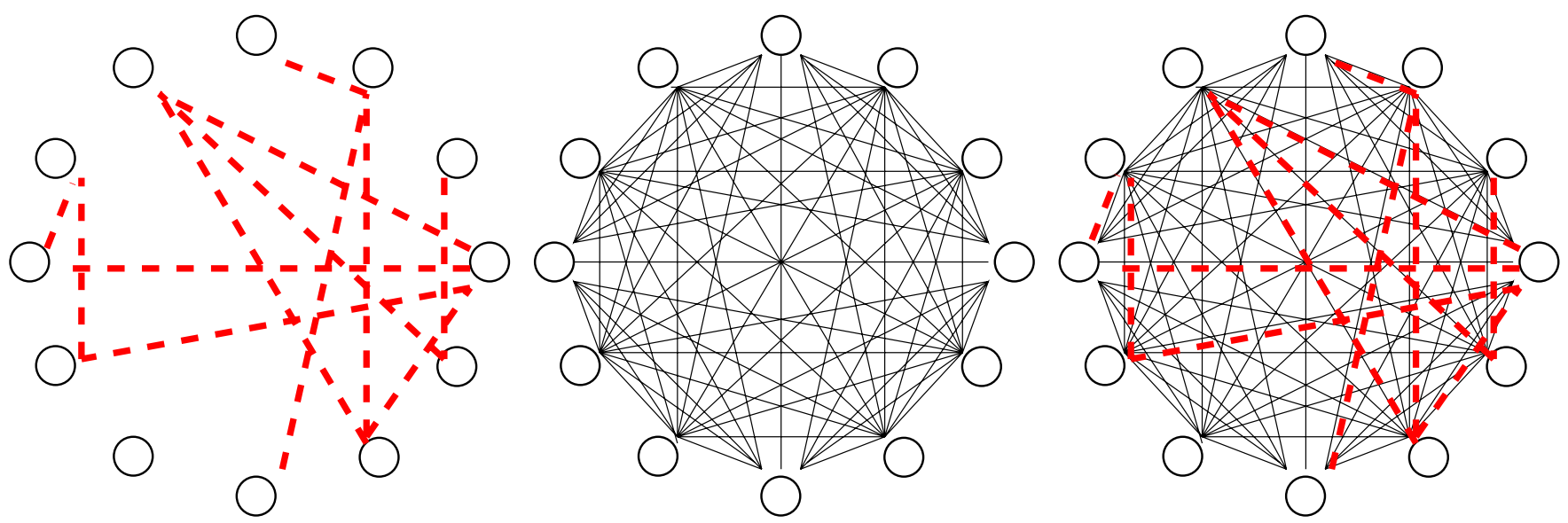

FIG. 2. (Color online) Left figure: a sparse model defined by some mean connectivity describes couplings in the sparse model. Center figure: a fully connected model describes couplings in the dense model. Right figure: a fully connected graph with a subset of strong sparse links; this is the composite model. The sparse subset of couplings is much stronger than the couplings on the other edges. 
The main question investigated in this paper is how phase behavior and transitions differ in the composite model from the behavior of the sparse and dense subsystems of which it is composed. Attention is restricted to cases above the percolation threshold in the random sparse substructure. In this way, the largest subset of variables connected through $O(1)$ (sparse) interactions is of size $O(N)$ and sufficient to allow a macroscopic ordering in the absence of dense interactions. For nonpercolating random graphs, with a largest connected component of size $O[\log (N)]$ or finite, it is thought the thermodynamic properties will be quantitatively similar to models of dense interactions among microscopic states. Other scalings may include substructures of intermediate size $\left[O\left(N^{\delta}\right), 0<\delta<1\right]$, as in nearly percolating substructures, which may allow distinct phenomena from both the above cases at low temperature.

Section II outlines the ensemble of models studied, which are then analyzed by the replica method in Sec. III. Section IV develops the replica equilibrium solution by population dynamics alongside an analogous belief propagation (BP) algorithmic method applicable to typical samples. A stability analysis of the BP equations and population dynamics is derived. Section V presents a leading-order solution to the composite system in terms of a simplified ansatz on the order parameter with results discussed in Sec. VI. Section VII demonstrates the RS solutions for several composite models in the interesting range of parameters about the triple point in the phase diagram. Section VIII presents hypotheses on the structure of the low-temperature phases alongside experimental results derived by BP and Monte Carlo methods. We then conclude with a brief summary.

\section{COMPOSITE ENSEMBLES}

The composite model can be described by a Hamiltonian with coupling of $N$ spins

$$
\mathcal{H}(\vec{S})=-\sum_{\langle i j\rangle}\left[J_{\langle i j\rangle}^{D}+J_{\langle i j\rangle}^{S}\right] S_{i} S_{j}-\sum_{i} z_{i} S_{i},
$$

where $\langle i j\rangle$ are an ordered set of variables. The couplings are labeled as dense $(D)$ or sparse $(S)$ and are sampled independently for each link according independent ensembles described shortly. The quenched variable abbreviation $\mathcal{Q}$ indicates a sample of the couplings and $\vec{S}$ are the dynamic variables. The field vector $\vec{z}$ is used only as a conjugate parameter to explore symmetries, the limit $\vec{z} \rightarrow \overrightarrow{0}$ (vector of zero fields) is always assumed throughout this chapter, although some physical quantities and insight are demonstrated using conjugate fields as described in Appendix B.

The equilibrium properties of the model are studied. The Hamiltonian implies a static probability distribution on the state space given by

$$
P(\vec{S})=\frac{1}{Z(\beta, \mathcal{Q})} \exp \{-\beta \mathcal{H}(\vec{S})\}
$$

where $\beta$ is the inverse temperature and $Z$ is the partition function.

The spin states of interest are the typical case equilibrium distribution, in the large system limit. Properties of these states are established through the mean free energy

$$
\beta f_{\mathcal{E}}(\beta)=-\lim _{N \rightarrow \infty} \frac{1}{N}\langle\log Z\rangle_{\mathcal{Q}},
$$

where $\mathcal{E}$ is the ensemble parametrization.

The model is fundamentally a fully connected one, the sparse component is realized as a subset of couplings that are $O(1)$ in magnitude, whereas all other couplings are weaker and rescaled to system size (decreasing as a function of $N$ ). Due to this difference in scaling with system size, many thermodynamic results for standard densely connected spin models do not apply.

\section{A. Dense (SK) substructure}

The dense substructure fully connects $N$ spin variables $\vec{S}$ $\in\{ \pm 1\}^{N}$, with couplings sampled independently and at random according to the Gaussian distribution parametrized by $J_{0}$ and $J$

$$
\begin{gathered}
P\left(J^{D}\right)=\prod_{\langle i j\rangle} P\left(J_{\langle i j\rangle}^{D}\right) ; \\
P\left(J_{\langle i j\rangle}^{D}\right)=\frac{1}{\sqrt{2 \pi J^{2} / N}} \exp \left\{-\frac{N}{2 J^{2}}\left(J_{\langle i j\rangle}^{D}-\frac{J_{0}}{N}\right)\right\},
\end{gathered}
$$

with a necessary scaling of components included. This set of couplings has a statistical description corresponding to the SK model.

\section{B. Sparse (VB) substructure}

It is convenient to factorize the sparse couplings as

$$
J_{\langle i j\rangle}^{S}=A_{\langle i j\rangle} V_{\langle i j\rangle} .
$$

The ensemble is described by a connectivity matrix $\mathrm{A}$, which is zero for all but a fraction $C / N$ of components, and a dense coupling matrix $\mathrm{V}$, with no zero elements. In the irregular ensemble, each directed edge is present (nonzero) independently with probability $C / N$, with $C$ as the mean variable connectivity, so that a prior for inclusion of an edge is

$$
P(\mathrm{~A})=\prod_{\langle i j\rangle}\left[\left(1-\frac{C}{N}\right) \delta\left(A_{\langle i j\rangle}\right)+\frac{C}{N} \delta\left(A_{\langle i j\rangle}-1\right)\right],
$$

this being the connectivity in a standard Erdös-Rényi random graph. The couplings in the nonzero cases are described by a distribution with finite moments and are sampled independently according to

$$
P(\mathrm{~V})=\prod_{\langle i j\rangle} P\left(V_{\langle i j\rangle}\right) ; \quad P\left(V_{\langle i j\rangle}=x\right)=\phi(x),
$$

in the general case. A practical distribution for analysis is the $\pm J$ distribution defined as

$$
\phi(x)=(1-p) \delta\left(x-J^{S}\right)+p \delta\left(x+J^{S}\right)
$$

with two parameters, $p$ is the probability that the link is antiferromagnetic, and $J^{S}$ is the strength of coupling. Regular 
connectivity ensembles have each variable constrained to interact with exactly $C$ neighbors,

$$
P(\mathrm{~A}) \propto \prod_{i=1}^{N} \delta\left(\sum_{j} A_{i j}-C\right) .
$$

\section{Representative parametrizations}

Four models are considered in greater detail owing to their simplicity and ability to make transparent a range of observed phenomena. The F-AF model includes ferromagnetic dense couplings $\left[J=0, J_{0}>0\right.$ [Eq. (4)]] and antiferromagnetic sparse couplings $[p=1$ [Eq. (8)]], with connectivity $C=2$, and is described by

$$
\mathcal{H}(\vec{S})=-\frac{\mathbb{B}\left(\gamma, J^{S}\right)}{N} \sum_{\langle i j\rangle} S_{i} S_{j}+J^{S} \sum_{\langle i j\rangle} A_{\langle i j\rangle} S_{i} S_{j} .
$$

The function $\mathbb{B}\left(\gamma, J^{S}\right) / N$ is introduced to balance the ferromagnetic and antiferromagnetic tendencies. Choosing $\mathbb{B}\left(\gamma, J^{S}\right)$ as a positive monotonically increasing function of the scalar parameter $\gamma$, the relative strength of the antiferromagnetic and ferromagnetic parts is kept in some intuitive balance. As $\gamma$ increases, there is an increased tendency toward aligning spins within the Hamiltonian-the ferromagnetic (ordered) state is promoted.

It is also interesting to consider the converse case, the AF-F model with a ferromagnetic sparse part $(p=0)$ and antiferromagnetic dense model $\left(J=0, J_{0}<0\right)$, with connectivity $C=2$,

$$
\mathcal{H}(\vec{S})=-J_{S} \sum_{\langle i j\rangle} A_{\langle i j\rangle} S_{i} S_{j}+\frac{\mathbb{B}\left(\gamma, J^{S}\right)}{N} \sum_{\langle i j\rangle} S_{i} S_{j},
$$

with $\mathrm{B}$ being again some suitably rescaled function, $J^{S}$ must also be defined.

These models can also be considered for the case of regular connectivity. Either antiferromagnetic couplings, the regular F-AF (10) and AF-F (11) models, are considered; but in each case with connectivity chosen to be $C=3$ (a minimal choice above the percolation threshold).

\section{REPLICA METHOD}

The replica method is used in both $[13,15]$ to study the composite system free energy in the limit of large $N$. The replica method is the most concise analytical method available, although many results presented herein can be developed through the cavity method with suitable assumptions. For convenience the fields $\vec{z} \rightarrow 0$, as in Eq. (1), in the various calculation steps. Variations in this are useful in establishing a number of system properties as outlined in Appendix B.

In the replica approach, the typical case behavior is examined through the free-energy density (3) averaged over the quenched disorder. That is to say we do not expect typical samples from the ensembles to differ in the value of the order parameters and other extensive properties. The replica identity

$$
\langle\log Z\rangle_{\mathcal{Q}}=\left.\frac{\partial}{\partial n}\right|_{n=0}\left\langle Z^{n}\right\rangle_{\mathcal{Q}}
$$

allows for the average over the logarithm to be replaced by the partition sum of a replicated set of variables. This is by an analytic continuation of $n$ to the set of integers, giving a form for which the quenched averages may be taken. The properties of the free energy are constructed through the replicated partition function

$$
\left\langle Z^{n}\right\rangle_{\mathcal{Q}}=\prod_{\alpha=1}^{n}\left(\sum_{\vec{S}^{\alpha}}\right)\left\langle\prod_{\langle i j\rangle} \exp \left\{\beta\left(J_{\langle i j\rangle}^{D}+J_{\langle i j\rangle}^{S}\right) \sum_{\alpha} S_{i}^{\alpha} S_{j}^{\alpha}\right\}\right\rangle_{\mathcal{Q}},
$$

where the quenched averages and dynamic averages may be taken equivalently.

The exponent is factorized with respect to the quenched variables in the sparse and dense parts. The average in the dense part involves an expansion to second order in $N$ of $J_{\langle i j\rangle}^{D}$. The leading-order terms are described by $J_{0}$ and $J^{2}$ [Eq. (4)], and higher-order terms are taken to be negligible in the large $N$ limit. The average in the sparse part is more involved, the full method is presented in Appendix A. The brief outline of the method in the remainder of this section applies only for Poissonian connectivity in the sparse substructure. The site dependence in the energetic part is factorized in general by introducing three classes of order parameters,

$$
q_{\alpha}=\frac{1}{N} \sum_{i} S_{i}^{\alpha} ; \quad q_{\left\langle\alpha_{1}, \alpha_{2}\right\rangle}=\frac{1}{N} \sum_{i} S_{i}^{\alpha_{1}} S_{i}^{\alpha_{2}} ; \quad \Phi(S)=\frac{1}{N} \sum_{i} \delta_{S, S_{i}},
$$

where $q_{\alpha}$ describes the homogeneous magnetization, $q_{\left\langle\alpha_{1}, \alpha_{2}\right\rangle}$ describes the two-replica correlations, and the generalized order parameter $[16] \Phi(S)$ describes many kinds of spin correlations, where the bold font vector notation is used to represent a vector labeled by replica indices rather than site indices, denoted by an over-line vector notation,

$$
\delta_{S, S_{i}}=\prod_{\alpha=1}^{n} \delta_{S^{\alpha}, S_{i}^{\alpha}} ; \quad S=\left\{S^{\alpha} \mid \alpha=1, \ldots, n\right\} .
$$

The order parameters $q_{\alpha}$ and $q_{\left\langle\alpha_{1}, \alpha_{2}\right\rangle}$ can be defined from the generalized order parameter in the Poissonian connectivity case

$$
q_{\alpha}=\sum_{\boldsymbol{\sigma}} \Phi(\vec{\sigma}) \sigma^{\alpha} ; \quad q_{\left\langle\alpha_{1}, \alpha_{2}\right\rangle}=\sum_{\boldsymbol{\sigma}} \Phi(\vec{\sigma}) \sigma^{\alpha_{1}} \sigma^{\alpha_{2}} .
$$

However, solving the saddle-point equations, by population dynamics in the RS description, where order parameters are assumed to be invariant under replica-index permutations, is complicated without the redundant description (14), and the redundant description is necessary in the regular and F-F models. Furthermore, having order parameters describing both dense and sparse parts is useful in discriminating effects due to sparse and dense substructures and the connection with the standard sparse and dense descriptions is also made transparent in the limiting cases: taking $q_{\alpha}=q_{\left\langle\alpha_{1}, \alpha_{2}\right\rangle}=0$ to re- 
cover the thermodynamics of a sparse system and $\Phi(\boldsymbol{\sigma})=1$ to recover a purely dense thermodynamic description.

The original mixed topology problem is replaced by a site factorized (mean field) model-the complexity being encoded in a set of interactions among replica encoded in the order parameters. The definitions of the order parameters may be transformed to an exponential form by introducing a weighted integral over conjugate parameters (denoted by a hat). The exponential form allows a saddle-point method to be applied; an extremization of the exponent allows the free energy to be identified as

$$
\begin{aligned}
\beta f_{\mathcal{E}}= & \lim _{n \rightarrow 0} \frac{\partial}{\partial n} \operatorname{Extr}_{\left\{\Phi, \hat{\Phi}_{, q_{\alpha}}, \hat{q}_{\alpha}, q_{\left\langle\alpha_{1}, \alpha_{2}\right.}, \hat{q}_{\left\langle\alpha_{1}, \alpha_{2}\right\rangle}\right\}}\left\{\mathcal{G}_{1}(\beta, \mathcal{E}, \Phi)\right. \\
& \left.+\mathcal{G}_{2}(\beta, \mathcal{E}, \hat{\Phi})+\mathcal{G}_{3}(\hat{\Phi}, \Phi)\right\},
\end{aligned}
$$

up to constant (ensemble parameter dependent) terms. The term $\mathcal{G}_{1}$ encodes an energetic term describing interactions, which in the absence of an external field is given by

$$
\begin{aligned}
\mathcal{G}_{1}= & -\frac{1}{2} \beta J_{0} \sum_{\alpha}\left(q_{\alpha}\right)^{2}-\frac{1}{2} \beta^{2} J^{2} \sum_{\left\langle\alpha_{1}, \alpha_{2}\right\rangle}\left(q_{\left\langle\alpha_{1}, \alpha_{2}\right\rangle}\right)^{2} \\
& -\frac{C}{2} \log _{S, S^{\prime}} \Phi(S) \Phi\left(S^{\prime}\right) \int d x \phi(x) \exp \left\{\beta x \sum_{\alpha} S^{\alpha} S^{\prime \alpha}\right\},
\end{aligned}
$$

where $\phi(x)$ is the coupling distribution in the sparse part (8). The term $\mathcal{G}_{2}$ is an entropic term coupling the sparse and dense order parameters

$$
\mathcal{G}_{2}=-\log \sum_{S} \exp \left\{\sum_{\alpha} \hat{q}_{\alpha} S_{\alpha}+\sum_{\left\langle\alpha_{1}, \alpha_{2}\right\rangle} \hat{q}_{\left\langle\alpha_{1}, \alpha_{2}\right\rangle} S^{\alpha_{1}} S^{\alpha_{2}}+C \hat{\Phi}(\boldsymbol{S})\right\} \text {. }
$$

The coupling between the order parameters and their conjugate forms is present in the term

$$
\mathcal{G}_{3}=C \sum_{S} \Phi(\boldsymbol{S}) \hat{\Phi}(\boldsymbol{S})+\sum_{\alpha} q_{\alpha} \hat{q}_{\alpha}+\sum_{\left\langle\alpha_{1}, \alpha_{2}\right\rangle} q_{\left\langle\alpha_{1}, \alpha_{2}\right\rangle} \hat{q}_{\left\langle\alpha_{1}, \alpha_{2}\right\rangle} .
$$

The free energy is used to calculate various self-averaging properties of the system by taking derivatives with respect to conjugate parameter, as outlined in Appendix B. The inverse temperature is conjugate to the energy, from which the entropy is calculated. Derivatives with respect to uniform fields conjugate to $\overrightarrow{1}$ can be used to test emergent ferromagnetic order. By inclusion of a random field of mean zero, the variance can be used to calculate correlation functions and susceptibility.

The order parameters defined at the extrema of the saddle point (denoted by $*$ ) obey coupled saddle-point equations,

$\Phi^{*}(\boldsymbol{S})=\mathcal{P}(\boldsymbol{S}) ; \quad q_{\alpha}^{*}=\sum_{\boldsymbol{S}} S^{\alpha} \mathcal{P}(\boldsymbol{S}) ; \quad q_{\left\langle\alpha_{1}, \alpha_{2}\right\rangle}^{*}=\sum_{\boldsymbol{S}} S^{\alpha_{1}} S^{\alpha_{2}} \mathcal{P}(\boldsymbol{S})$,

$$
\mathcal{P}(\boldsymbol{\sigma}) \propto \exp \left\{C \hat{\Phi}^{*}(\boldsymbol{\sigma})+\sum_{\alpha} \hat{q}_{\alpha}^{*} \sigma^{\alpha}+\sum_{\left\langle\alpha_{1}, \alpha_{2}\right\rangle} \hat{q}_{\left\langle\alpha_{1}, \alpha_{2}\right\rangle}^{*} \sigma^{\alpha_{1}} \sigma^{\alpha_{2}}\right\},
$$

is a normalized probability distribution on the replicated state space.

The conjugate parameters are determined by equations without coupling between the sparse and dense parts

$$
\begin{gathered}
\hat{\Phi}^{*}(\vec{\sigma}) \propto \sum_{\tau} \Phi^{*}(\tau)\left\langle\exp \left\{\beta x \sum_{\alpha} \tau^{\alpha} \sigma^{\alpha}\right\}\right\rangle_{x} ; \\
\hat{q}_{\alpha}^{*}=\beta J_{0} q_{\alpha}^{*} ; \quad \hat{q}_{\left\langle\alpha_{1}, \alpha_{2}\right\rangle}^{*}=\beta^{2} J^{2} q_{\left\langle\alpha_{1}, \alpha_{2}\right\rangle}^{*},
\end{gathered}
$$

with $x$ distributed according to $\phi(x)$ as in Eq. (7). From these six equations, it is possible to eliminate the conjugate parameters [Eq. (23)] to leave a fixed point defined without the conjugate parameters.

\section{REPLICA SYMMETRIC FORMULATION AND BELIEF PROPAGATION}

\section{A. RS saddle-point equations}

The order parameters are defined by the standard sparse and dense RS forms

$$
\Phi^{*}(\vec{\sigma})=\int d h \pi(h) \prod_{\alpha=1}^{n} \frac{\exp \left\{h \sigma^{\alpha}\right\}}{2 \cosh h} ; \quad q_{\alpha}^{*}=m ; \quad q_{\left\langle\alpha_{1}, \alpha_{2}\right\rangle}^{*}=q,
$$

with the variational aspects captured by the normalized distribution on the real line $(\pi)$ and two scalar parameters $(m, q)$.

The saddle-point equations can then be written for the general case, inclusive of regular and Poissonian connectivity, as

$$
\pi(h) \propto \int\left\langle\prod_{c=1}^{c_{e}}\left[d h_{c} d x_{c} \pi\left(h_{c}\right) \phi\left(x_{c}\right)\right] \delta\left(h-h^{R S}\right)\right\rangle_{c_{e}, \lambda},
$$

where

$$
h^{R S}=m+\lambda \sqrt{q}+\sum_{c=1}^{c_{e}} \operatorname{atanh}\left[\tanh \left(\beta x_{c}\right) \tanh \left(h_{c}\right)\right],
$$

and $c_{e}$ is distributed according to the excess connectivity distribution, a normalized distribution proportional to $C P(C-1)$, where $P(C)$ is the full variable connectivity distribution, regular or Poissonian. The integration variable $\lambda$ is normally distributed. The dense parts are defined similarly

$$
m=\int\left\langle\prod_{c=1}^{c_{f}}\left[d h_{c} d x_{c} \pi\left(h_{c}\right) \phi\left(x_{c}\right)\right] \delta\left(h-h^{R S}\right) \tanh (h)\right\rangle_{c_{f}, \lambda},
$$

where

and 


$$
q=\int\left\langle\prod_{c=1}^{c_{f}}\left[d h_{c} d x_{c} \pi\left(h_{c}\right) \phi\left(x_{c}\right)\right] \delta\left(h-h^{R S}\right) \tanh ^{2}(h)\right\rangle_{c_{f}, \lambda},
$$

but with the averages in $c_{f}$ being with respect to the full connectivity distribution.

These equations can be solved numerically by a method of population dynamics [17] subject to two additional recursions on scalar quantities (27) and (28).

\section{B. Composite belief propagation equations}

Composite BP can be interpreted in the context of the composite system as a heuristic method of determining marginals of the static probability distribution (40) given a quenched sample [18]. Whereas an exhaustive calculation requires $O\left(2^{N}\right)$ operations to construct a marginal, $\mathrm{BP}$ is guaranteed to produce an estimate in a number of operations that scales only linearly with the number of edges.

The equations from factors to nodes are trivial in the case of binary factors, so iterations on variable messages alone can be composed. Defining two directed messages for every link $\langle i j\rangle$, which can be interpreted as log-posterior ratios for spins on graphs with some interactions removed (cavity graphs)

$$
\begin{aligned}
& h_{i \rightarrow j}^{(t+1)}=\frac{1}{2 \beta} \sum_{\tau_{i}} \tau_{i} \log \hat{P}^{(t+1)}\left(S_{i}=\tau_{i} \mid G_{i \rightarrow j}\right) \\
& =\frac{1}{\beta} \sum_{k\{\{i, j\}} \operatorname{atanh}\left[\tanh \left(\beta h_{k \rightarrow i}^{(t)}\right) \tanh \left(\beta J_{\langle i k\rangle}\right)\right],
\end{aligned}
$$

where $\hat{P}$ is used to denote an approximated probability distribution. The cavity graph is a factor graph rooted in variable $i$ with the coupling $J_{\langle i j\rangle}$ set to zero. The assumption underlying the probabilistic recursion is the independence of log-posterior ratios, which allows them to be used accurately as priors in each step, so that the recursion is equivalent to that on a tree.

$\mathrm{BP}$ can be iterated from some initial condition. If correlations between messages are sufficiently weak then the messages will converge to correctly describe the probabilities. Marginal properties, such as the magnetization at equilibrium, can be constructed from converged messages. A log marginal may be estimated by

$$
\begin{aligned}
H_{i}^{(t+1)} & =\frac{1}{2 \beta} \sum_{\tau} \tau \log \hat{P}^{(t+1)}\left(S_{j}=\tau \mid G\right) \\
& =\frac{1}{\beta} \sum_{j \backslash i} \operatorname{atanh}\left[\tanh \left(\beta h_{j \rightarrow i}^{(t)}\right) \tanh \left(\beta J_{\langle i j\rangle}\right)\right] .
\end{aligned}
$$

The condition of sufficiently weak correlations is closely related to the notion of a pure state is statistical mechanics [2]. The assumption of independent messages applies only when the log posteriors [Eq. (29)] reflect the distribution in a pure state, the similarity with Eq. (26) is not coincidental. Pure states act as local attractors of the BP dynamics, and it is only when there is a competition between these attractors that dynamics is expected to fail. With BP initialized sufficiently close (globally) to a pure state, or in the case of a unique attractor, convergence to the pure state can be anticipated giving a correct description of the equilibrium probability distribution.

\section{Simplification of dense messages}

Assuming the messages to be independent then each message can be considered as a random object determined by the couplings in the cavity graph. The messages are therefore iid and the sum over many messages will converge to a Gaussian random variable. To leading order, the messages may be rewritten incorporating this insight

$$
\begin{aligned}
h_{i \rightarrow j}^{(t+1)}= & m^{(t)}+\sqrt{q^{(t)}} \lambda_{i \rightarrow j}^{(t)} \\
& +\frac{1}{\beta} \sum_{\left.k \in\left\{\partial_{i} \backslash\right\}\right\}} \operatorname{atanh}\left[\tanh \left(\beta h_{k \rightarrow i}^{(t)}\right) \tanh \left(\beta J_{\langle i j\rangle}\right)\right],
\end{aligned}
$$

where $m^{(t)}$ is the mean and $q^{(t)}$ is the variance, and term $\partial_{i}$ is used to denote variables connected to $i$ through strong couplings. The distribution over reweighted messages $\lambda_{i \rightarrow j}$ will be asymptotically Gaussian if the approximation is correct. The value of the message for a particular instance of the quenched disorder is given by

$$
m^{(t)}+q^{(t)} \lambda_{i \rightarrow j}^{(t)}=\frac{1}{\beta} \sum_{k \backslash\left\{\partial_{i} \cup j\right\}} \operatorname{atanh}\left[\tanh \left(\beta h_{k \rightarrow i}^{(t)}\right) \tanh \left(\beta J_{\langle i j\rangle}\right)\right] .
$$

The Gaussian statistics are defined by analogy with the RS thermodynamic quantities, to leading order in $N$

$$
m^{(t)}=\beta J_{0} \frac{1}{N} \sum_{i=1}^{N} \tanh \left(\beta H_{i}^{(t)}\right) ; \quad q^{(t)}=\beta^{2} J^{2} \frac{1}{N} \sum_{i=1}^{N} \tanh ^{2}\left(\beta H_{i}^{(t)}\right),
$$

for any dense set of couplings [19]. The log-posterior ratios for the spin states on the full graph are approximated as

$\beta H_{j}^{(t+1)}=m^{(t)}+\sqrt{q^{(t)}} \lambda_{j}^{(t)}+\sum_{k \in \partial_{i}} \operatorname{atanh}\left[\tanh \left(\beta h_{k \rightarrow i}^{(t)}\right) \tanh \left(\beta J_{\langle i j\rangle}\right)\right]$.

The term $\lambda_{i}$ is closely related to $\lambda_{i \rightarrow j}$, up to a correction on the order of $1 / N$, by removing the restriction on the sum in $j$ from Eq. (32).

In the case that $J \neq 0$ it is necessary to evaluate $\lambda_{i}$ for each link, still requiring $O\left(N^{2}\right)$ evaluations as in the original algorithm. To reduce computational complexity, it may be valuable to marginalize over this if $J \ll J_{0}$ or if the sparse couplings dominate dynamics, but if $J=0$ it is sufficient to take $\lambda_{i}^{(t)}=0$ and algorithm complexity is reduced to $O(N)$, as illustrated in Fig. 3. A method for combining messages in models comprising both densely and sparsely interacting components has been recently introduced [20].

\section{Stability analysis}

If the replica description correctly describes a single pure state, then this implies that the spin-glass susceptibility is not 

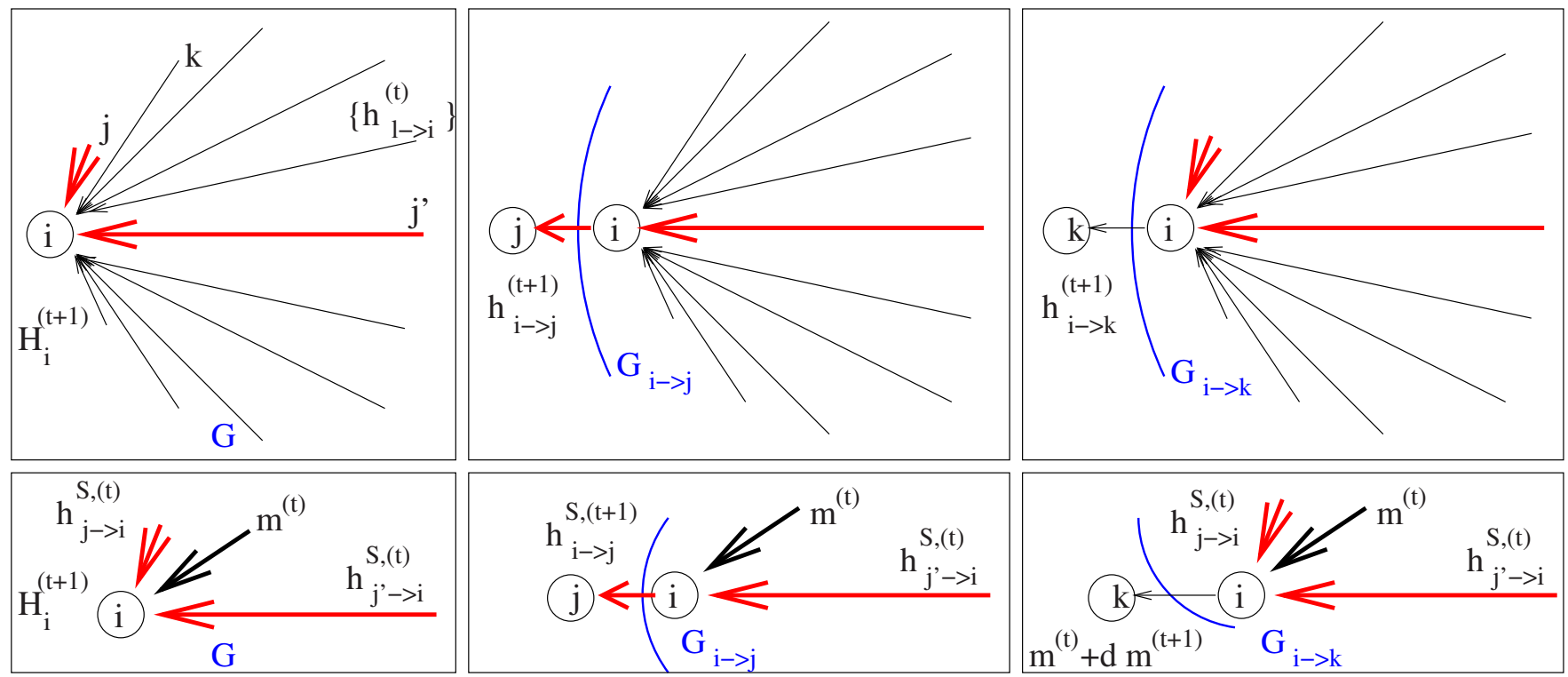

FIG. 3. (Color online) BP constructs an estimate of the posteriors by message passing, each message is a log-posterior estimate for some variable subject to the removal of the interaction with another variable (the variable to which the message is passed), as in the top subfigures. In the lower two subfigures, the central limit is applied to the messages on dense links and in some cases only a single parameter is then required to represent the $O(N)$ dense messages. A related approximation is implicit in the derivation of the RS free energy.

divergent in the thermodynamic limit. In the case of a sparse graph, the treelike approximation provides a natural basis for constructing a self-consistent estimate of the spin-glass susceptibility [21]; whereas in the dense model a direct test of eigenvalue stability toward replica symmetry breaking can establish a complete description [22].

An analytic framework entirely within the replica method might be constructed to test spin-glass susceptibility. As in Appendix B, a connection can be made between the particular instability in the order parameter and the divergence of the physical quantity, spin-glass stability, within the RS framework. This identity is not pursued within this paper, instead a more intuitive framework, believed to be equivalent, is presented.

The nondivergence of the spin-glass susceptibility in sparse and dense models requires the local stability of the saddle-point equations; this proves to be an equivalent condition to the stability of the BP equations on a typical graph in the limit $N \rightarrow \infty[19,21]$. Stability of the BP equations is therefore explored for a typical sample. Assuming a linear perturbation $\left\{\delta h_{i \rightarrow j}^{(t)}\right\}$ about some fixed point $\left\{h_{i \rightarrow j}^{(t)}\right\}$ of the BP Eq. (29) implies an independent recursion on the perturbations that may be written at the leading order,

$$
\delta h_{j \rightarrow k}^{(t+1)}=\sum_{i \backslash\{j, k\}} \delta h_{i \rightarrow j}^{(t)} \frac{\left[1-\tanh ^{2}\left(\beta h_{i \rightarrow j}^{(t)}\right)\right] \tanh \left(\beta J_{\langle i j\rangle}\right)}{1-\tanh ^{2}\left(\beta h_{i \rightarrow j}^{(t)}\right) \tanh ^{2}\left(\beta J_{\langle i j\rangle}\right)} .
$$

In the dense part, the fluctuations may again be represented by a Gaussian random variable of mean and variance

$$
\begin{gathered}
J_{0}\left\langle\delta h_{i \rightarrow j}^{(t)}\left[1-\tanh ^{2}\left(\beta h_{i \rightarrow j}^{(t)}\right)\right]\right\rangle \\
\left\langle\left(\delta h_{i \rightarrow j}^{(t)}\right)^{2}\left[1-\tanh ^{2}\left(\beta h_{i \rightarrow j}^{(t)}\right)\right]^{2}\right\rangle,
\end{gathered}
$$

respectively, since the couplings are assumed to be uncorrelated with the perturbations in BP, the average is with respect to all perturbations and fields incident on $j$. An expansion of $h_{i \rightarrow j}$ in terms of $H_{i}$ is possible so that the statistics can be shown to be identical at leading order for all $j$ [19]; therefore, the perturbations evolve according to quantities which are time but not site dependent,

$$
\begin{gathered}
\delta m^{(t)}=J_{0}\left\langle\delta H_{i}^{(t)}\left[1-\tanh ^{2}\left(\beta H_{i}^{(t)}\right)\right]\right\rangle ; \\
\delta q^{(t)}=J^{2}\left\langle\left(\delta H_{i}^{(t)}\right)^{2}\left[1-\tanh ^{2}\left(\beta H_{i}^{(t)}\right)\right]^{2}\right\rangle,
\end{gathered}
$$

where $\delta H_{i}^{(t)}$ are the perturbations in the log posteriors, which are equal to $\delta h_{i \rightarrow j}^{(t)}$ at the leading order whenever $J_{\langle i j\rangle}$ is not a strong-coupling term.

A final approximation is to assume $H_{i}$ is uncorrelated with $\delta H_{i}$. In this case, the statistics can be written only in terms of $q^{(t)},\left\langle\delta H_{i}\right\rangle$, and $\left\langle\left(\delta H_{i}\right)^{2}\right\rangle$. However, this is not true at leading order when a sparse component is present. Variables with larger connectivity in the sparse part are described by a field distribution of greater variance, and the perturbations scale similarly. Instead, the pair of correlation functions [Eq. (37)] determines the evolution of perturbations.

Evolution of the perturbations can be undertaken in parallel with BP; to each message is attached a representative statistic for, or a distribution over, perturbations. It is sufficient to consider a distribution of perturbations characterized by a mean $\delta \bar{h}_{i \rightarrow j}^{(t)}$ and variance $\delta \bar{h}_{i \rightarrow j}^{2(t)}$ attached to each macroscopic field. If these parameters decay exponentially, in expectation, then this is an indication of fixed-point stability. 


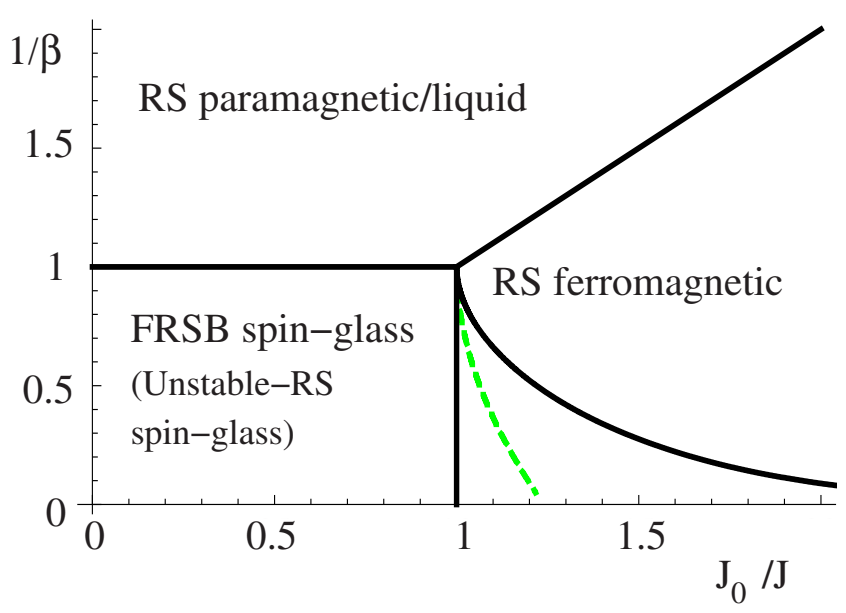

NEAR TRIPLE POINT

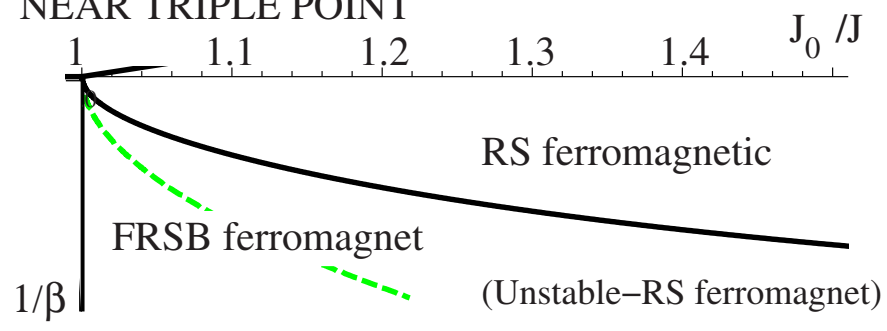

(Unstable-RS spin-glass)

FIG. 4. (Color online) The phase diagrams for disordered spin-glass systems often exhibit a phase behavior similar to the SK model. Left figure: the phase transitions are indicated by solid dark lines. As temperature is lowered, there is a transition from an RS paramagnetic phase $(m=q=0)$ to either an RS ferromagnetic $(m>0)$ or spin-glass $(q>0, m=0)$ phase. As temperature is lowered in the ferromagnetic phase, there is also an RS to full-RSB transition. Under the RS assumption, the longitudinal instability measures calculated in the context of BP coincides with the F-SG transition in the RS description (dashed line). The instability of the ferromagnetic phase is not correctly predicted; the result is a lower bound in temperature for the replica instability in the ferromagnetic phase (toward a mixed phase).

Assuming that there is no linear instability, the equation determining $\delta \bar{h}_{i \rightarrow j}^{2(t)}$ is

$$
\delta \bar{h}_{i \rightarrow j}^{2(t)}=\delta q^{(t)}+\sum_{i \in \partial_{j} \backslash k} \delta \bar{h}_{i \rightarrow j}^{2(t)}\left(\frac{\left[1-\tanh ^{2}\left(\beta h_{i \rightarrow j}^{(t)}\right)\right] \tanh \left(\beta J_{\langle i j\rangle}\right)}{1-\tanh ^{2}\left(\beta h_{i \rightarrow j}^{(t)}\right) \tanh ^{2}\left(\beta J_{\langle i j\rangle}\right)}\right)^{2},
$$

with a similar equation applicable to the case of a linear perturbation.

The BP equations can be interpreted as a recursive instantiation of the RS saddle-point Eqs. (25)-(28) except in the explicit site dependence, so that quenched disorder specific correlations may accumulate over several updates. Assuming a negligible feedback process in BP, or a modified problem without loops or with annealed disorder, the macroscopic properties established by BP will depend only on the steadystate distribution of messages on sparse links and the mean and variance of dense messages. Objects analogous to a histogram estimate to $\pi$ [Eq. (25)] and scalar parameters $m^{(t)}$ and $q^{(t)}$ in the saddle-point method. However, at the level of the mapping of individual points in the RS description (26), it is possible that local fluctuations of the messages on fields are unstable, despite stability in the distribution. Whereas divergence in $\langle\overrightarrow{\delta h}\rangle$ might be observed in a macroscopic instability in the first moment of $\pi$, an instability of the mapping in $\left\langle\delta \bar{h}^{2}\right\rangle$ will not be realized in any macroscopic moment of the distribution. It is this instability in the mapping which is probed by the BP stability analysis. In the absence of a linear instability, it is assumed that divergence in $\left\langle\delta \bar{h}^{2}\right\rangle$ is a necessary condition for any local instability.

The fluctuations on sparse messages are represented fully in this framework, whereas dense messages are summarized under approximation. The stability is a self-consistent (longitudinal) test of stability but is known not to probe all possible instabilities and so provides only a sufficient criteria for instability. The SK model is an example where the longitudinal stability of the ferromagnetic phase, as derived through a BP framework [19], does not capture correctly the spinglass transition at low temperature, as shown in Fig. 4. Since the models investigated in detail later have inhomogeneity in the sparse substructure only $\left(J^{2}=0\right)$, it is felt that the test of stability, as applied in this paper, may be a more accurate reflection of true local stability toward replica symmetry breaking. A connection between the stability tested through the BP framework and an instability entirely within the replica method might be established as outlined in Appendix B.

\section{EXACT HIGH-TEMPERATURE FORMULATION}

In the limit $\beta \rightarrow 0$, the paramagnetic solution $\Phi=1, q_{\alpha}$ $=0, q_{\left\langle\alpha_{1}, \alpha_{2}\right\rangle}=0$ is the only stable solution but becomes unstable as temperature is decreased. This process can be investigated by considering the moments of $\Phi$ through a moment expansion representation

$$
\begin{aligned}
\Phi(\boldsymbol{\sigma})= & 1+\sum_{\alpha} \bar{q}_{\alpha} \sigma^{\alpha}+\sum_{\left\langle\alpha_{1}, \alpha_{2}\right\rangle} \bar{q}_{\left\langle\alpha_{1}, \alpha_{2}\right\rangle} \sigma^{\alpha_{1}} \sigma^{\alpha_{2}} \\
& +\sum_{L=3} \sum_{\left\langle\alpha_{1}, \ldots, \alpha_{L}\right\rangle} \bar{q}_{\left\langle\alpha_{1}, \ldots, \alpha_{L}\right\rangle} \sigma^{\alpha_{1}} \ldots \sigma^{\alpha_{L}} .
\end{aligned}
$$

The saddle-point equations can be solved in each moment $\{\bar{q}\}$ and stability tested in some subset of the moments.

In the sparse substructure, both the excess and full connectivity distributions are Poissonian, the saddle-point Eq. (21) can be expanded, using the identity (16), as

$$
\begin{aligned}
P(\boldsymbol{\sigma})= & \prod_{L=1}^{\infty}\left\{\prod _ { \langle \alpha _ { 1 } , \ldots , \alpha _ { L } \rangle } \left[\cosh \left(X_{L} \bar{q}_{\left\langle\alpha_{1}, \ldots, \alpha_{L}\right\rangle}\right)\right.\right. \\
& \left.\times\left(1+\sigma^{\alpha_{1}} \cdots \sigma^{\alpha_{L}} \tanh \left(X_{L} \bar{q}_{\left\langle\alpha_{1}, \ldots, \alpha_{L}\right\rangle}\right)\right]\right\},
\end{aligned}
$$

eliminating the conjugate parameters [Eq. (23)]. The terms 


$$
X_{1}=\beta J_{0}+T_{1} ; \quad X_{2}=\beta^{2} J^{2}+T_{2} ; \quad X_{i}=T_{i} \text { if } i>2,
$$

determine transition properties, where

$$
T_{i}=C \int d x \phi(x) \tanh ^{i}(\beta x) .
$$

The saddle-point equations can be written in terms of an equation for each moment

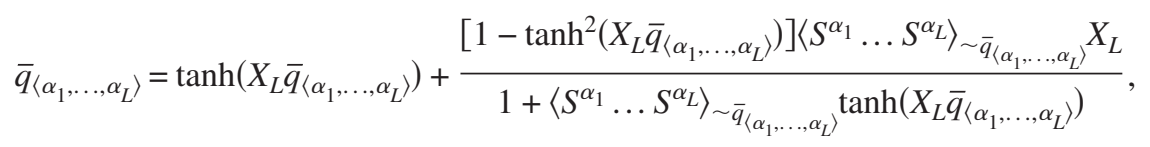

where the notation $\langle\cdots\rangle_{\sim x}$ indicates an average with respect to Eq. (40), but with $x=0$. A solution is apparent, which is the paramagnetic solution with $z=\left\langle\sigma^{\alpha_{1}} \ldots \sigma^{\alpha_{L}}\right\rangle$ and $q_{\left\langle\alpha_{1}, \ldots, \alpha_{L}\right\rangle}=0$ for all choices of indices. This is the only solution when $X_{L} \rightarrow 0$, corresponding to the high-temperature limit.

At lower temperature, a solution may emerge in one of the moments. It is only necessary to show that some component $\bar{q}$ allows a nonzero solution. The second term in Eq. (43) is zero at leading order in $\bar{q}$ in the moments of the distribution, and there is no coupling of the moments at leading order. Hence, any solution which emerges continuously from the paramagnetic solution must do so with equality at leading order between the first term of the right-hand side and the left-hand side. This leads to criteria $X_{L}=1$ for the existence of a continuous transition.

For a discontinuous transition to occur in some component, without $X_{i}>1$, requires the derivative of the second part with respect to $\bar{q}$ to be a convex function of $\bar{q}$ in some range of the parameter (43). However, the derivative is a concave function of $\bar{q}$, so that unless $X_{i}>1$ for some component, there can be no solution other than the paramagnetic one.

\section{A. High-temperature phase transitions}

The existence of nonparamagnetic order is determined from Eq. (43) as

$$
\begin{gathered}
X_{1}>1 \quad \text { 1-spin,ferromagnetic(F)order, } \\
X_{2}>1 \quad \text { 2-spin, spin-glass(SG)order, } \\
X_{L}>1 \quad L \text {-spin order. }
\end{gathered}
$$

In each case, the solution which emerges may be estimated by an expansion in the right-hand side of Eq. (43) up to some order. Cubic order can be considered as a minimum to obtain the continuously emerging solution. To allow for the replica limit $n \rightarrow 0$, an assumption on the correlations is required, RS being the simplest, and the order parameters may then be determined. Depending on the order of solution required, some coupling between moments is relevant, and it is necessary to solve a set of coupled equations.
The emergence of a ferromagnetic phase is realized in a continuous transition toward nonzero values of $\bar{q}_{\alpha}$. Through coupling of the order parameters, all parameters $\bar{q}_{\left\langle\alpha_{1}, \ldots, \alpha_{L}\right\rangle}$ become nonzero at order $O\left[\left(\bar{q}_{\alpha}\right)^{L}\right]$.

The emergence of a spin-glass phase is realized in a continuous transition toward nonzero values of $\bar{q}_{\left\langle\alpha_{1}, \alpha_{2}\right\rangle}$, while $\bar{q}_{\alpha}=0$. Even order parameters are generated by the coupling of terms at a higher order.

The transition toward an $L$-spin order is irrelevant to the high-temperature analysis, since by consideration of Eq. (41) it is clear that $X_{L} \leq X_{2}$ for all $L>2$, with equality only in pathological cases, therefore, the transition can only be toward a ferromagnetic or spin-glass phase.

In the case that $X_{1}=X_{2}$, at the high-temperature transition point both orders may emerge simultaneously and in competition. This case can be understood at leading order through an SK auxiliary model.

\section{B. SK auxiliary system}

In either the case of a ferromagnetic order, or spin-glass order, the behavior is described at leading order about the paramagnetic phase by the terms $\left\{q_{\alpha}\right\}$ and $\left\{q_{\left\langle\alpha_{1}, \alpha_{2}\right\rangle}\right\}$. The free energy can be written in these cases as a function of only these two types of order parameter. After elimination of conjugate parameters, the free energy can be written up to constant terms as

$$
\begin{aligned}
\beta f_{\mathcal{E}}= & \lim _{n \rightarrow 0} \frac{\partial}{\partial n}\left(-\log \sum_{S} \exp \left\{X_{1} \sum_{\alpha} q_{\alpha} S^{\alpha}\right.\right. \\
& \left.+X_{2} \sum_{\left\langle\alpha_{1}, \alpha_{2}\right\rangle} q_{\left\langle\alpha_{1}, \alpha_{2}\right\rangle} S^{\alpha_{1}} S^{\alpha_{2}}\right\}+\frac{X_{1}}{2} \sum_{\alpha} q_{\alpha}^{2} \\
& \left.+\frac{X_{2}}{2} \sum_{\left\langle\alpha_{1}, \alpha_{2}\right\rangle} q_{\left\langle\alpha_{1}, \alpha_{2}\right\rangle}^{2}\right) .
\end{aligned}
$$

This is the replica formulation of the SK model free energy [7]. Therefore, at leading order the high-temperature phases are equivalent to the SK model, up to the $\beta$ dependence of the energetic coupling terms. Instead of the standard term $\beta J_{0}$, there is $X_{1}$, and instead of $\beta^{2} J^{2}$ there is $X_{2}$.

For every composite system of Poissonian connectivity, there exists an auxiliary SK model with an equivalent 
leading-order behavior at high temperature. By mapping the composite parametrizations to the SK model, all the leadingorder high-temperature transition properties must carry over, including the nature of replica symmetry breaking (RSB) and the stability of the RS description.

Let $A$ denote the parametrizations $\left(J_{0}^{A}, J^{A}, \beta^{A}\right)$ of an SK model with an equivalent high-temperature behavior to some composite system at the high-temperature transition. This parametrization is redundant, there are only two independent parameters, and so $J^{A}=1$ is chosen. The standard phase diagram for an SK model under this parametrization is demonstrated in Fig. 4.

The auxiliary parametrization is determined by the mapping equilibrating the coefficients in the free energy (41),

$$
\beta^{A} J_{0}^{A}=X_{1} ; \quad\left(\beta^{A}\right)^{2}=X_{2} .
$$

Where this mapping is continuous, it is possible to consider how the auxiliary system parametrization responds to variation in temperature (or some other parameter) in the composite system. Variation in $\beta$ in the composite model is realized as a trajectory in the auxiliary model parameter space given by

$$
\frac{\partial J_{0}^{A}}{\partial \beta^{A}}=2 \frac{J_{0}-J_{S} C\left[1-\tanh ^{2}\left(\beta J^{S}\right)\right]}{J^{S} C \tanh \left(\beta J^{S}\right)\left[1-\tanh ^{2}\left(\beta J^{S}\right)\right]}-\frac{1}{\beta^{A}} .
$$

In the case that the couplings to higher-order moments are small $\left(X_{L} \ll 1\right.$ for $L>2$ ) then the mapping may be applied with some confidence to lower temperature. Such a scenario will occur when the $X_{1}$ and $X_{2}$ are dominated by the dense substructure terms, or when $C$ is large in the sparse substructure.

\section{Beyond leading order}

The leading-order approximation to the composite system differs from the SK model in the anomalous dependence of energetic components on $\beta$. This observation alone is sufficient to account for many of the novel features of composite models reported at high temperature.

About the ferromagnetic transition, the term $q_{\alpha}$ appears at leading order to provide a thermodynamic description. The magnitude of $\left(q_{\alpha}\right)^{2}$ is proportional to $\Delta_{1}=X_{1}-1$ at leading order and at $L^{\text {th }}$ order the value is dependent on moments of the distribution up to $\bar{q}_{\left\langle\alpha_{1}, \ldots, \alpha_{L}\right\rangle}$. The set of nonlinear coupled equations can be solved in parallel at each order. The ferromagnetic phase is at leading order an RS phase so that an expansion with simple RS components will be stable at leading order. The full description of the ferromagnetic phase differs from the auxiliary system description at the third or fourth order.

The spin-glass phase does not include any nonzero odd moments and is described at leading order by $\Delta_{2}=X_{2}-1$ and, at second order, includes the term $\bar{q}_{\left\langle\alpha_{1}, \alpha_{2}, \alpha_{3}, \alpha_{4}\right\rangle}$. This term arises from the sparse substructure and so behavior deviates from the auxiliary model at second order. However, since even moments have positive coefficients, all with a monotonic dependence on $\beta$, phenomenological properties may not differ significantly from the VB model, which has been thoroughly studied (e.g., [10]).
In the vicinity of the triple point, where both $\Delta_{1}$ and $\Delta_{2}$ are positive, the terms $\bar{q}_{\left\langle\alpha_{1}, \alpha_{2}, \alpha_{3}\right\rangle}$ and $\bar{q}_{\left\langle\alpha_{1}, \alpha_{2}, \alpha_{3}, \alpha_{4}\right\rangle}$ are relevant at the second order. The literature developed in studying the VB model is sufficient to describe RS properties and stability about the triple point [9,23]. The leading-order behavior gives a transition from an RS ferromagnet to a spin glass according to a balance in the components $\Delta_{1}=\Delta_{2} / 2$. The second-order term in the sparse model indicates the existence of a mixed phase, with a refinement of the transition line.

The de Almeida-Thouless (AT) line is sufficient to describe stability of an RS solution in the dense model at all temperatures [22]. In order to correctly describe transitions in the sparse or composite models, it is necessary to consider a wider range of eigenvalues [23], which cannot be evaluated other than numerically, except at the percolation threshold (absent in the composite model) or as a polynomial expansion truncated at some order.

A stability analysis considering moments up to fourth order was recently presented [15]. It considers an RS description with inclusion of non-zero $\left\{\bar{q}_{\left\langle\alpha_{1}, \alpha_{2}, \alpha_{3}\right\rangle}, \bar{q}_{\left\langle\alpha_{1}, \alpha_{2}, \alpha_{3}, \alpha_{4}\right\rangle}\right\}$ but with an analysis of instabilities restricted to variation in $\left\{\bar{q}_{\alpha}, \bar{q}_{\left\langle\alpha_{1}, \alpha_{2}\right\rangle}\right\}$. This predicts a comparable splitting of the line $\Delta_{1}=\Delta_{2} / 2$ to those found for the VB model, but for some ranges of parameters a stable spin-glass phase is incorrectly identified. Since only a restricted set of eigenvalues is considered, this is not unreasonable but demonstrates a weakness in the method.

\section{Regular connectivity}

The derivations of this section, so far, beginning from Eq. (43) onward have been specific to the case of Poissonian connectivity (19) and do not necessarily extend to composite models with non-Poissonian connectivity. The replica theory is developed along similar lines to previous sections in Appendix $A$ to be inclusive of the regular connectivity ensemble. The 1-spin and 2-spin dense substructure order parameters are determined by Eq. (14) and take zero values in the paramagnetic phase. The sparse substructure order parameter is different from Eq. (14) to be inclusive of nonPoissonian connectivity but in general takes a value $\Phi=1$ in the paramagnetic solution and may be expanded as a set of moments [Eq. (39)]. However, with the new definition $q_{a}$ $\neq \bar{q}_{\alpha}$ and $q_{\left\langle\alpha_{1}, \alpha_{2}\right\rangle} \neq \bar{q}_{\left\langle\alpha_{1}, \alpha_{2}\right\rangle}$ in general. Each of these order parameters corresponds to distinct physical quantities: $q_{\alpha}\left(q_{\left\langle\alpha_{1}, \alpha_{2}\right\rangle}\right)$ are related to the mean magnetization (2-spin correlation), whereas $\bar{q}_{\alpha}, \bar{q}_{\left\langle\alpha_{1}, \alpha_{2}\right\rangle}$ correspond to these quantities weighted by connectivity in the sparse substructure, as indicated in Appendix B.

Along similar lines to the previous analysis, it is possible to consider the emergence of order by treatment only of the leading-order behavior about the paramagnetic solution. The 1 -spin order terms are coupled at leading order by the saddlepoint equations; thus there is no decoupled representation describing emergence of spin glass and ferromagnetic order in general. The criteria for a ferromagnetic solution to emerge continuously from the paramagnetic solution as temperature are lowered is determined by the point at which 


$$
\left(\begin{array}{l}
q_{\alpha} \\
\bar{q}_{\alpha}
\end{array}\right)=\left(\begin{array}{cc}
\beta J_{0} & T_{1} \tanh (\beta x) \\
\beta J_{0} & \frac{(C-1)}{C} T_{1}
\end{array}\right)\left(\begin{array}{l}
q_{\alpha} \\
\bar{q}_{\alpha}
\end{array}\right),
$$

if such a point exists; its existence requires the principal eigenvector of the matrix to be the one vector. However, the existence of a solution point in the coupled equations is not guaranteed, and there exist a range of parameters in which decreasing temperature results in a pair of complexconjugate eigenvalues, which exceed one in modulus.

The right-hand side of Eq. (48) represents the leadingorder 1-spin terms in the saddle-point Eq. (21), after elimination of the conjugate parameters. In the case of Poissonian connectivity, the existence of a continuous transition is necessary for the existence of a ferromagnetic or spin-glass phase (43). This is due to the concavity of the saddle-point equation when interpreted as a mapping, concavity is assumed to hold also for the regular connectivity composite system.

However, in a general model it is necessary only for the principal eigenvalue of the matrix (48) to exceed one for some nonparamagnetic solution of the saddle-point equations to exist. When the modulus of the principal eigenvalue exceeds one, the assumption of weak coupling between the moments in the order parameters expansion ceases to be valid when considering an expansion coincident with the eigenvector. The criteria that the modulus in the leading-order expansion are greater than one corresponds to a set of criteria,

$$
\frac{1}{2}\left|\left(\beta J_{0}+\frac{C-1}{C} T_{1}\right) \pm \sqrt{\left(\beta J_{0}+\frac{C-1}{C} T_{1}\right)^{2}+4 \frac{\beta J_{0} T_{1}}{C}}\right|
$$

$>1$ 1-spin order,

$$
\begin{aligned}
& \frac{1}{2}\left|\left(\beta^{2} J^{2}+\frac{C-1}{C} T_{2}\right) \pm \sqrt{\left(\beta^{2} J^{2}+\frac{C-1}{C} T_{2}\right)^{2}+4 \frac{\beta^{2} J^{2} T_{2}}{C}}\right| \\
& \quad>1 \text { 2-spin order, }
\end{aligned}
$$

$$
\frac{C-1}{C} T_{L}>1 \quad L \text {-spin order. }
$$

The potential exists for the modulus to exceed one, while the discriminant is less than zero in the 1-spin order term, when either $T_{1}$ or $\beta J_{0}$ is negative. This phenomena, absent in the VB and SK models, is contingent on a subset of couplings being antiferromagnetic. In spite of a comparable functional form in the 2-spin order term, the transition from a paramagnet to a spin glass is always described by a non-negative discriminant and real eigenvalues.

The complex eigenvalues imply complex-conjugate eigenvectors. Where the eigenvalues are real, it is possible to test the stability of the equilibrium solution by inclusion of a conjugate field in proportion to the eigenvector components (see Appendix B). However, where the eigenvalue is complex such a field is not physical and is not consistent with assumptions made in the development of the equilibrium solution.
Attention is restricted to real-valued perturbations of the order parameters, which can be associated with the realvalued conjugate fields. A local instability in the paramagnetic solution is only anticipated toward a ferromagnetic phase when the real part of the principal eigenvalue is larger than one or toward a spin-glass solution when criteria [Eq. (49)] are met. If the paramagnetic solution is stable with respect to an infinitesimal term conjugate to the magnetization in the Hamiltonian then the paramagnetic solution will be recovered continuously as the conjugate field approaches zero. This is equivalent to the criteria that the linearized saddle-point equations are convergent to the zero solution. Linear instability is apparent when the real part of the eigenvalues exceeds one. However, since the perturbation is not coincident with an eigenvector there is no leading-order solution to the linearized equations when the external field is added. The instability in the paramagnetic solution is toward a discontinuously emerging solution.

The discontinuously emerging solution from the paramagnetic instability might be a locally stable (thermodynamic or metastable) solution across a wider range of temperatures than that indicated by the local stability analysis of the paramagnetic solution. In limited simulations, comparable in size to those described in Sec. VIII, the behavior observed at temperatures close to (but below) the modulus one criteria (49) is consistent with the hypothesis of two locally stable solutions. One solution describes the thermodynamic phase, and the other a metastable solution, with decreasing temperature a discontinuous thermodynamic is anticipated.

The case of large $\gamma$ allows only for a transition from a paramagnetic to ferromagnetic state, and this may be discontinuous. As well as a thermodynamic solution, several dynamical transitions may describe changes in local stability criteria of the solutions; these local instabilities may be dominate aspects of dynamics, and in general will not be coincident with thermodynamic transitions.

At intermediate $\gamma$ values, the paramagnetic solution may be locally unstable first toward a spin-glass solution as temperature is lowered. The presence of another metastable or thermodynamic ferromagnetic phase may change the properties of this transition by comparison with the standard continuous case.

In the limit of large $C$, a simplified description is possible in the transition criteria for the regular connectivity case. With a sensible scaling of the moments of $\phi(x)$ so that $T_{1}$ and $T_{2}$ remain finite as $C$ increases, the final term in the discriminant (49) becomes negligible and a simple transition criteria is recovered, consistent with the Poissonian system

$$
\beta J_{0}+T_{1}>1 ; \quad \beta^{2} J^{2}+T_{2}>1
$$

This is also the result that would be obtained in naively applying the dense system method, using only a mean and variance of link strengths, to the two-scale system. Examples of discontinuous high-temperature transitions are examined in Sec. VI C, with a clear departure from the conditions laid out in Eq. (50). 


\section{LEADING-ORDER PREDICTIONS FOR PHASE BEHAVIOR}

\section{A. F-AF model}

The SK auxiliary model can be used to predict trends as temperature or some other parameter is varied in the F-AF model about the high-temperature transition points. Using the mapping (46) combined with an exact (full-RSB) description of the transitions and phases of the SK model at high and low temperatures, the trajectories implied by the mapping can be used as a leading-order predictor of phase behavior.

Choosing the F-AF models [Eq. (10)] such that

$$
\mathbb{B}=\gamma ; \quad J^{S}=\operatorname{atanh}(1 / \sqrt{C}),
$$

a class of models parametrized by $\gamma \in[0, \infty)$ is created. The disorder in couplings decreases with $\gamma$ from a typical spin glass set to an ordered ferromagnetic set. These models are characterized by a high-temperature spin-glass transition at $\beta_{C}=1$ when $\gamma<1$, and a high-temperature ferromagnetic transition at a temperature $\beta_{C}^{-1}=\gamma$ when $\gamma>1$. There is a triple point in the parameter space at $\gamma=1, \beta=1$. Phase transitions between ferromagnetic and spin-glass phases are possible where $\beta \gtrsim 1$ and $\gamma \sim 1$.

Near the triple-point model parametrization $(\gamma=1)$, a decrease in temperature results in a competition between ferromagnetic and spin-glass solutions. A graphical answer to which solution dominates is provided by Fig. 5, for a range of high-temperature transition properties. If only leadingorder moments are considered in the free energy then all composite systems evolve toward an RS ferromagnetic behavior with decreasing temperature. Thus, unusual transitions away from FRSB spin-glass phases toward RS ferromagnetic phases are predicted as temperature is lowered.

The auxiliary model is an approximation except very close to the high-temperature transition, where higher-order moments are negligible and linear approximations apply. At lower temperature, complicated couplings with these higherorder moments may prevent an FRSB to RS transition. However, working at the level of linearization near the transition point the unusual FRSB to RS transitions are still observed in some models.

In the F-AF model, a spin-glass phase with zero magnetization cannot be a sufficient description at low temperature. This is because the spins disconnected from the sparse substructure can evolve independently and undergo an independent phase transition induced by the dense substructure. The results at the leading order are in agreement with this observation.

\section{B. AF-F model}

Consider the assignments

$$
\mathbb{B}=\gamma\left[1-C \tanh \left(J_{S} / \gamma\right)\right], \quad J^{S}=\operatorname{atanh}(1 / \sqrt{C}),
$$

as applied to the AF-F model (11), with $\gamma$ $\in\left[0, J_{S} / \operatorname{atanh}(1 / C)\right]$ describing the level of order in couplings. Larger $\gamma$ can be considered, but these correspond to systems with small ferromagnetic couplings in the dense part rather than antiferromagnetic ones.

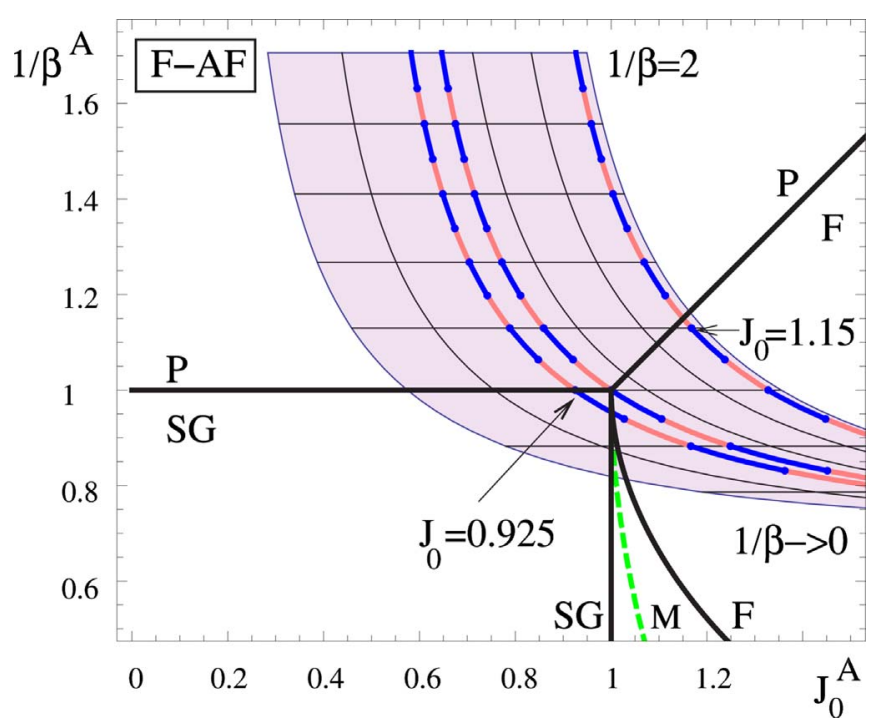

FIG. 5. (Color online) The F-AF models [Eq. (10)] in a parameter range $(\gamma=[0.75,1.25], 1 / \beta=(0,2])$ are mapped through Eq. (46) to auxiliary SK models parametrized by $\left(J_{0}^{A} / J^{A}, 1 / \beta^{A}\right)$. These models are equivalent about the high-temperature transition lines and elsewhere equivalent when constraining higher than secondorder moments to zero [Eq. (39)]. Horizontal isobars indicate constant $\beta$, and the near vertical isobars indicate constant $\gamma$, in the composite model parameter space. The set of transition lines for the SK model is shown; the upper most solid lines describing the hightemperature phase transition. The SK auxiliary model predicts that as temperature is lowered in the composite models, behavior converges toward a mean-field ferromagnetic behavior. For small $\gamma$, the prediction is that a spin-glass phase transforms through a mixed phase to an RS ferromagnet behavior as temperature is lowered. Decreasing temperature about the triple point $(\gamma=1)$, there is only an RS ferromagnetic behavior. The three highlighted isobars correspond to composite systems from left to right parametrized by $\gamma$ $=0.952\left(J_{0}^{A}=0.925\right.$ at $\left.\beta_{C}\right), \gamma=1\left(J_{0}^{A}=1\right.$ at $\left.\beta_{C}\right)$, and $\gamma=1.23\left(J_{0}^{A}\right.$ $=1.15$ at $\beta_{C}$ ), across a range of temperatures.

The predictions based on a leading-order representation of the order parameters are shown in Fig. 6. Composite systems are predicted to evolve toward spin-glass phases as temperature is lowered; lowering temperature at large $\gamma$ results first in transitions to stable RS ferromagnetic phases then toward a mixed phase before finally a spin-glass phase. The auxiliary model predicts that at lower temperature the magnetic moment is suppressed, for all $\gamma$ up to the maximum value $J_{S} / \operatorname{atanh}(1 / C)$, so that in the low-temperature limit all systems are in a phase equivalent to a "finite temperature" spinglass phase in the SK model. As temperature is lowered, RS states become unstable toward RSB, which is the scenario normally observed in dense or sparse spin-glass models.

The prediction that all systems converge toward a finite temperature spin glass is a consequence of the limited moment description. The spin-glass behavior is a residual effect of the sparse couplings and, at low temperature, depends strongly on higher-order moments, which are absent in the auxiliary model. The spin-glass phase is not induced by the dense antiferromagnetic couplings. 


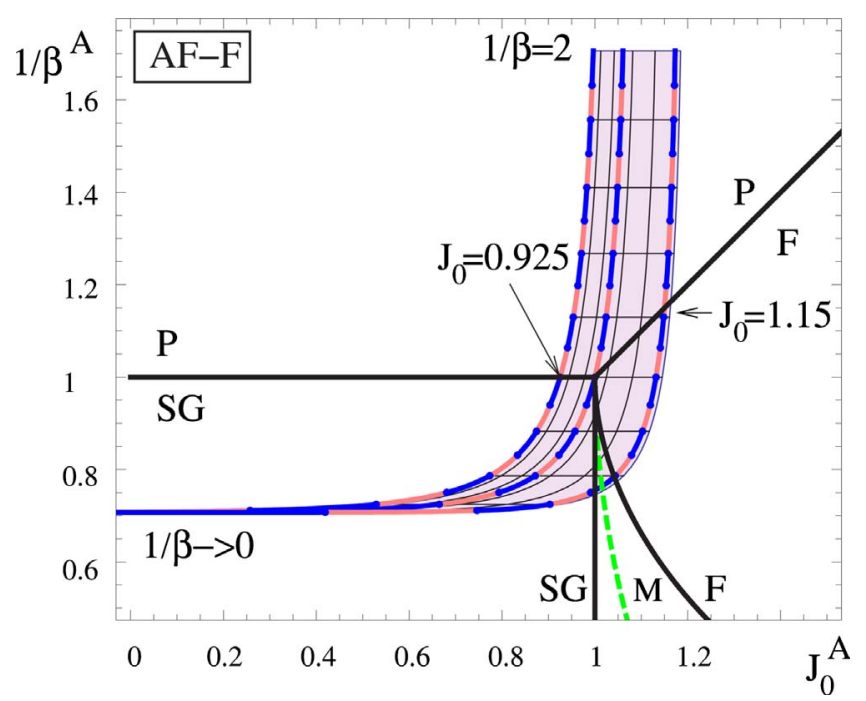

FIG. 6. (Color online) The AF-F model (11) as parametrized in $\gamma-\beta$ space $(\gamma=[0.75,1.25], 1 / \beta=[0,2])$ is mapped [Eq. (46)] to an auxiliary dense model parameter space. The auxiliary model prediction is that the magnetic order parameter $\left(\mathrm{m}^{2}\right)$ goes to zero in all composite models as temperature is lowered; a FRSB spin-glass phase describes the zero-temperature limit. The three highlighted systems correspond to systems with $\gamma=0.746\left(J_{0}^{A}=0.925\right.$ at $\left.\beta_{C}\right), \gamma$ $=1\left(J_{0}^{A}=1\right.$ at $\left.\beta_{C}\right)$, and $\gamma=1.23\left(J_{0}^{A}=1.15\right.$ at $\left.\beta_{C}\right)$.

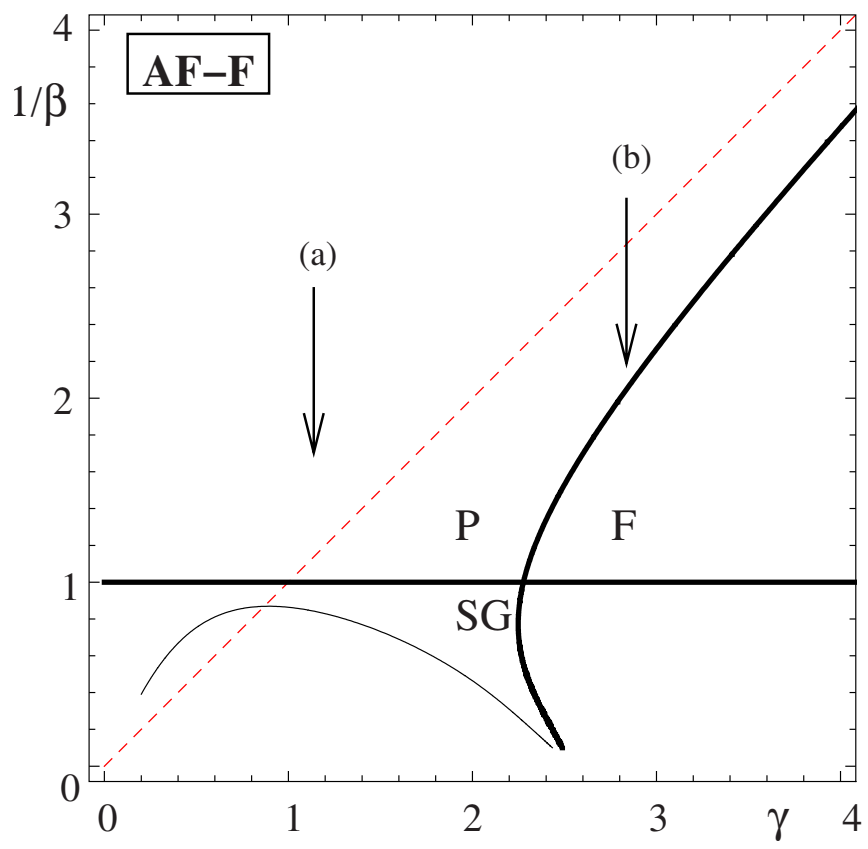

\section{Regular connectivity models}

Figure 7 demonstrates the limitations on the parameter range consistent with unique locally stable RS solutions, in the case of regular connectivity systems. The two figures correspond to the models (10) and (11), but with regular couplings [Eq. (9)] and mean connectivity 3 . The coupling scaling is

$$
\mathbb{B}=\gamma\left[1-C \tanh \left(J_{S} / \gamma\right)\right] ; \quad J^{S}=\operatorname{atanh}(1 / \sqrt{C-1}) .
$$

The choice of $J^{S}$ ensures that everywhere temperature $\beta=1$ corresponds to a spin-glass instability in the paramagnetic solution. The choice of scaling means that under the approximated ferromagnetic transition scheme (50), the critical temperature implying local instability in the paramagnetic solution toward ferromagnetism increases linearly with $\gamma$ denoted by the dashed line in Fig. 7. If the transition were predicted by Eq. (50) then a triple point would occur at 1: for $\gamma<1$ all high-temperature transitions would be of a spinglass type and for $\gamma>1$ transitions would be of a ferromagnetic type.

With couplings characterized by Eq. (53), a range of $\gamma$ values allow the instability of the paramagnetic solution toward 1-spin order to be described by complex eigenvalues. In the AF-F regular model, at small values of $\gamma$, the complex eigenvectors describe the stability of the paramagnetic solu-

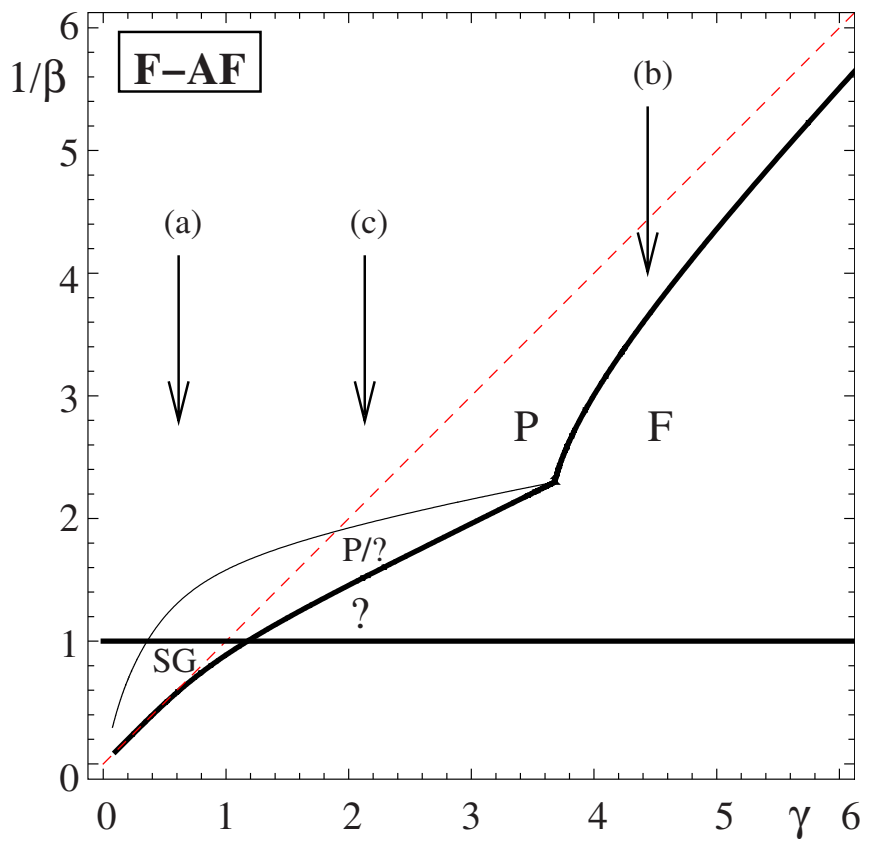

FIG. 7. (Color online) In this figure, the horizontal line indicates a high-temperature instability in the paramagnetic solution toward 2-spin order. Other lines indicate instabilities toward 1-spin order: the straight diagonal line is assuming the conditions [Eq. (50)], the thick and thin lines are the points where the real part or modulus of the principal eigenvector(s) equal one, respectively. Left figure: in the AF-F model, decreasing temperature results in either a continuous spin glass or ferromagnetic transition. (a) At small $\gamma$, a spin-glass phase emerges continuously with decreasing temperature. (b) At large $\gamma$, eigenvectors describing 1-spin order are real; a continuous ferromagnetic transition is found. Right figure: in the F-AF model, continuous and discontinuous transitions occur; no continuous transition triple-point exists. (a) At small $\gamma$, eigenvectors describing the 1-spin order are complex, but a spin-glass high-temperature transition is dominant. (b) At large $\gamma$, a continuous transition occurs described by a real eigenvector. (c) An instability in the paramagnetic solution in the first moment is anticipated at the lower (thick) line for intermediate $\gamma$, the properties of the discontinuously emerging solution (labeled ?) cannot be established by a linearized approach. The thin line indicates instability in the modulus for the linearized system, which is speculated to relate to the existence of the nonparamagnetic solution. 

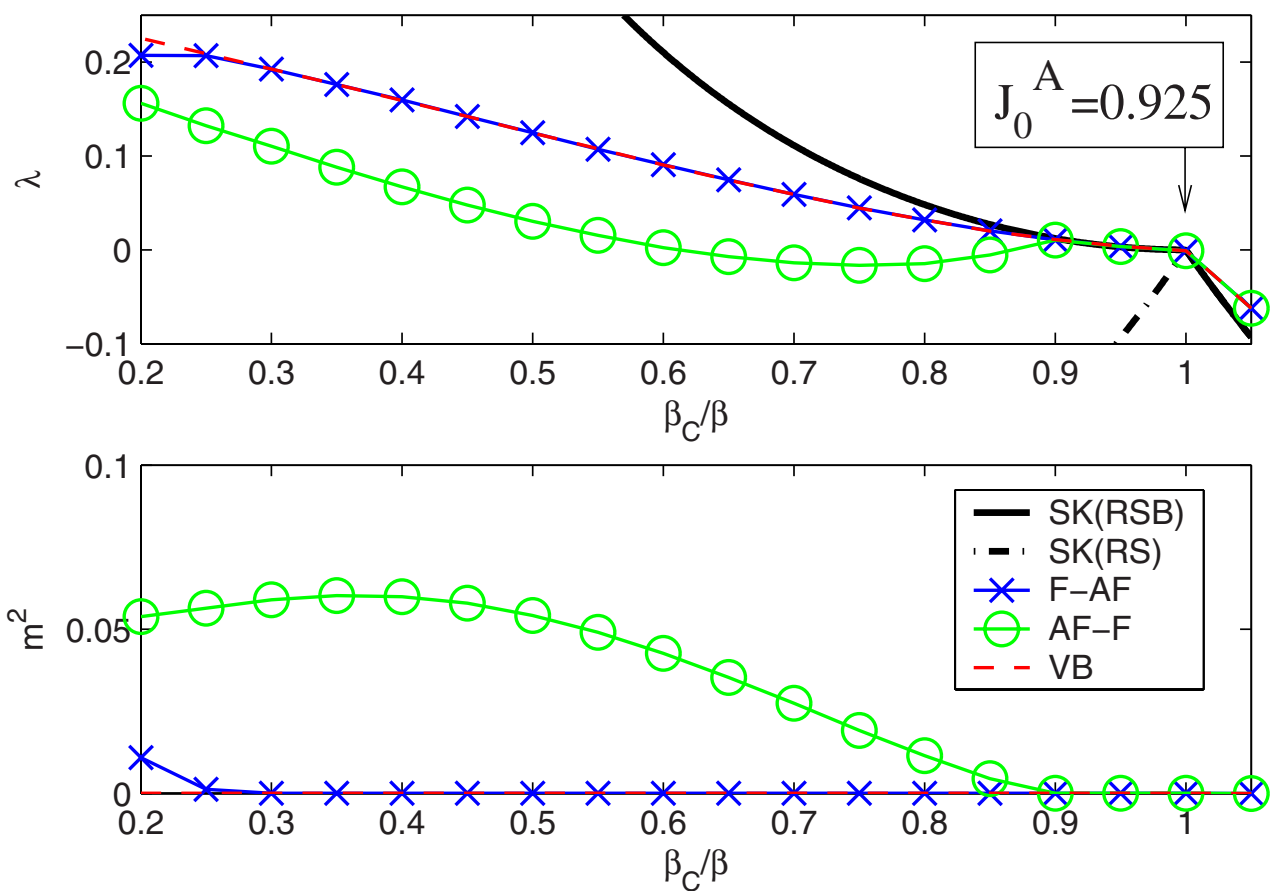

FIG. 8. (Color online) A comparison of the stability exponent and magnetization for the F-AF (circles), AF-F (crosses), VB (dashed line), and SK (solid line) models under the RS assumption. Every model is equivalent at the high-temperature spin-glass transition point to an SK model parametrized by $J_{0}^{A}=0.925$, and temperature variation is considered on the rescaled interval $\beta_{C} / \beta=[0.2,1.05]$. In the top figure, two stability exponents are given for the SK model, a longitudinal measure SK(RS) and a latitudinal measure SK(RSB). In the lower figure, the sparse and dense models show similar trends with $\lambda>0$ and $m^{2}=0$. Composite models behave as sparse spin-glass models whenever $m^{2}$ $=0$; but there is a departure in both models at low temperature. In all models as temperature decreases $\lambda>0$, except for the F-AF model, which is negative over an intermediate temperature range. Both composite models attain a nonzero magnetic moment at low temperature, which is not seen in the VB or SK models. The F-AF model is in approximate agreement with Fig. 5 at a high temperature. However, the behaviors observed in the composite models at low temperature are not anticipated by the auxiliary model.

tion toward 1-spin order; but as temperature is lowered a spin-glass instability is first attained. At larger $\gamma$ (equivalently $J_{0}$ ), a triple point is reached, but here the eigenvectors are real, and a continuous transition from the paramagnetic to ferromagnetic phase is found at larger $\gamma$.

In the F-AF regular model, complex eigenvalues occur in a parameter range relevant to the high-temperature transition. When $J_{0}$ is sufficiently large, a continuous high-temperature ferromagnetic transition is observed and, at small $\gamma$, there is a continuous spin-glass transition. There exists a broad range of $\gamma$ between these regimes where the ferromagnetic solution cannot emerge continuously from the paramagnetic solution and two locally stable solutions are anticipated. There is no triple point in this model suitable for a perturbative analysis.

In a small number of Metropolis-Hastings Monte Carlo simulations [24], two attractors corresponding to paramagnetic- and ferromagnetic-type configurations were found in these parameter ranges, though no systematic analysis was undertaken.

\section{REPLICA SYMMETRIC SOLUTION OF LOW-TEMPERATURE BEHAVIOR}

In Figs. 8-10, stability measures and magnetizations for the composite models, equivalent at $\beta_{C}$ to SK models with $J_{0}^{A}=1, J_{0}^{A}=1.15$, and $J_{0}^{A}=0.925$, are presented at various tem- peratures below the $1 / \beta_{C}$. The trends found are compared to those predicted by the auxiliary model in the vicinity of the transitions, as shown in Figs. 5 and 6, and also RS solutions to dense (SK) and sparse (VB) models with equivalent hightemperature properties.

\section{A. Numerical evaluation of the saddle-point equations}

To work beyond a perturbative approach, the RS saddlepoint equations are solved by population dynamics [17]. The results are presented based on samples from a single run of a population dynamics algorithm. In population dynamics, machine numbers are used for $m$ and $q$ and the distribution $\pi$ is represented by an order-parameter histogram $(W)$ of $N$ components

$$
\pi \rightarrow W=\left\{h_{1}, \ldots, h_{N}\right\} .
$$

The saddle-point Eqs. (25)-(28) are treated as a mapping with integrals and summations replaced by random samples. This implies a random map from the histogram to itself. Updating histograms recursively by a large number of random maps, from a random initial condition, leads to an accurate description of the fixed point $\pi$.

The random sampling is done in such a way as to reduce fluctuations in the variance of the Gaussian distributed samples and mean of the Poissonian distributed samples to 

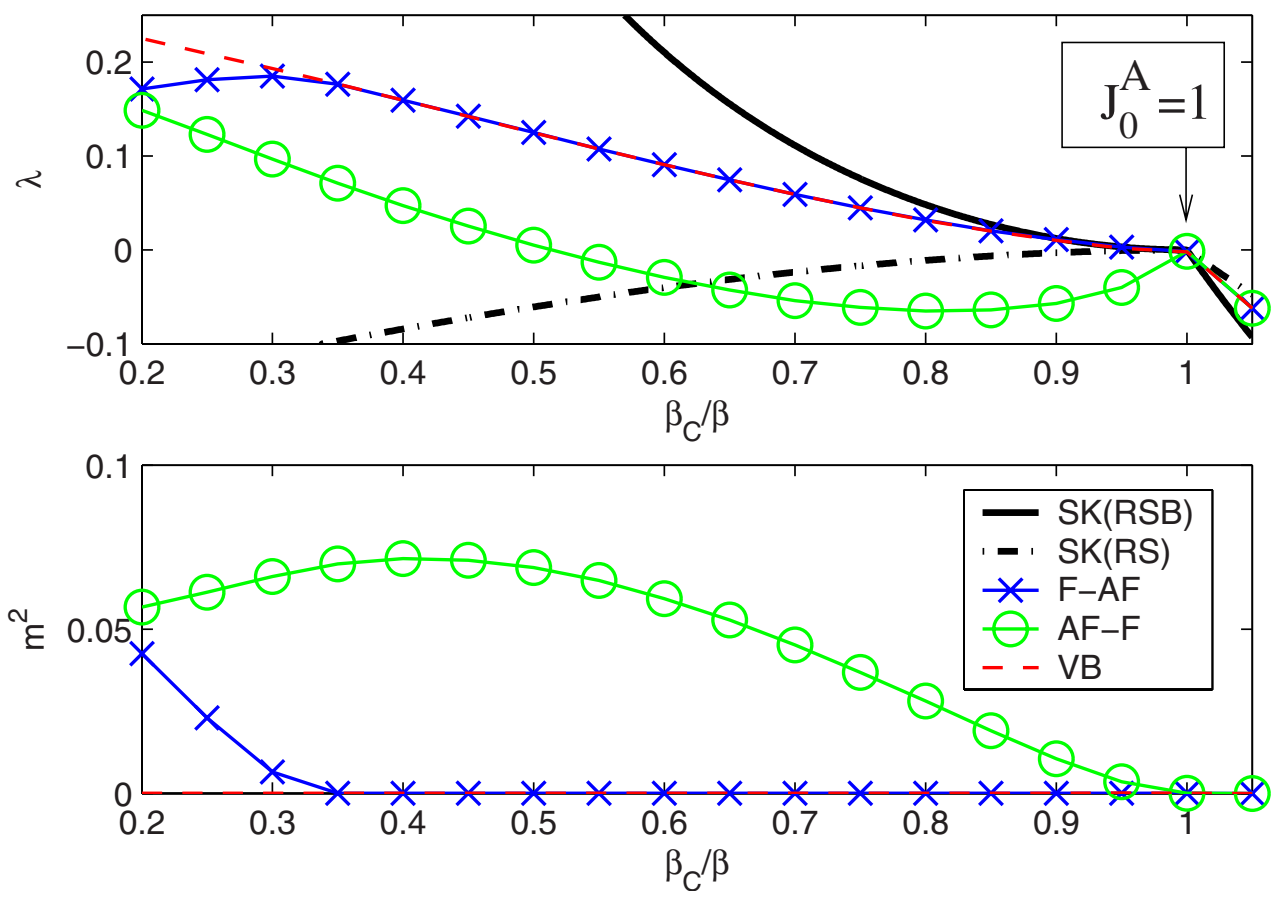

FIG. 9. (Color online) A comparison of the longitudinal stability and magnetization for the F-AF (circles), AF-F (crosses), VB (dashed line), and SK (solid line) models under the RS assumption. Every model is equivalent at the high-temperature transition to an SK model with $J_{0}^{A}=1$, coincident with the triple point in the phase diagram. Temperature variation is considered on the rescaled interval $\beta_{C} / \beta$ $=[0.2,1.05]$. Trends differ in F-AF from Fig. 5 in that the magnetization acquires a maximum value, and the stability exponent tends toward a positive value at sufficiently low temperatures. Trends differ in AF-F from Fig. 6 in the appearance of a magnetic moment at low temperatures.

$O(1 / N)$. A single iteration includes an update of every field in the histogram $W$ with either parallel or random sequential order. Given that antiferromagnetic couplings play a role in the dynamics, there is a risk that an invalid macroscopic antiferromagnetic state could be amplified by parallel updates. This scenario does not form a problematic point in the analysis undertaken but was relevant to work undertaken in [15] and carefully avoided. In order to control finite-size effects a scheme of microcanonical sampling was employed with respect to $W$, so that each field in generation $(t)$ is involved in forming exactly $C$ fields in generation $(t+1)$.

A histogram of 65556 floating point fields run for 1024 iterations appears to resolve all statistical quantities of interest down to a temperature of $\sim 1 /\left(10 \beta_{C}\right)$, with great precision, even in the vicinity of phase transitions. At lower temperature, there is a rapid decrease in the resolution of statistical quantities, which is uniform across tested systems and probably related to numerical precision limitations in the representation for hyperbolic functions. Based on the converged set of order parameters, samples are taken in the following 256 iterations to determine robust system statistics.

The initial condition for the order parameters $m^{2}, q$, and $W$ are chosen as paramagnetic combined with a small systematic bias toward spin-glass and ferromagnetic configurations with small but nonzero values to the dense substructure moments $\left(m^{2}=q\right)$, elements of $W$ are sampled according to a Gaussian $\mathcal{N}(m, q)$ such that the mean and variance of the histogram values are $m+O(1 / N)$ and $q=O(1 / N)$. Other initial conditions were also tested to ensure that dynamical bias was not implied by initial conditions; the suggested scheme converged effectively and systematically.

\section{Numerical evaluation of the stability equations}

The longitudinal stability is tested by initializing a fluctuation histogram $\delta W$

$$
\delta W=\left\{\left(\chi^{2}\right)_{1}^{(t)},\left(\chi^{2}\right)_{2}^{(t)}, \ldots,\left(\chi^{2}\right)_{N}^{(t)}\right\},
$$

where each component corresponds to a distinct field in the histogram $W$ (54). Each component represents a topology free measure of $\delta \bar{h}_{i \rightarrow j}^{2(t)}$, each of which is evolved according to Eq. (38), with the site-dependent fields and parameters replaced by a sample of fields from $W$ and other quenched disorder determined as in the field update. Cases in which $J^{2}=0\left(q^{(t)}=0\right)$, without linear perturbations are considered. The stability exponent is

$$
\lambda^{(t)}=\log \frac{\sum_{l}\left(\chi^{2}\right)_{l}^{(t)}}{\sum_{l}\left(\chi^{2}\right)_{l}^{(t-1)}},
$$

which is negative if BP is convergent in expectation. This is averaged over many generations, alongside renormalization of $\delta W$ to prevent numerical precision problems.

\section{B. F-AF and AF-F models}

Results for VB, SK, F-AF, and AF-F models are shown. The VB model presented for comparison is of connectivity 2, the same as the sparse substructures for F-AF and AF-F models, and has a balance of antiferromagnetic and ferromagnetic interactions described by a $\pm J$ model (8). Figures 

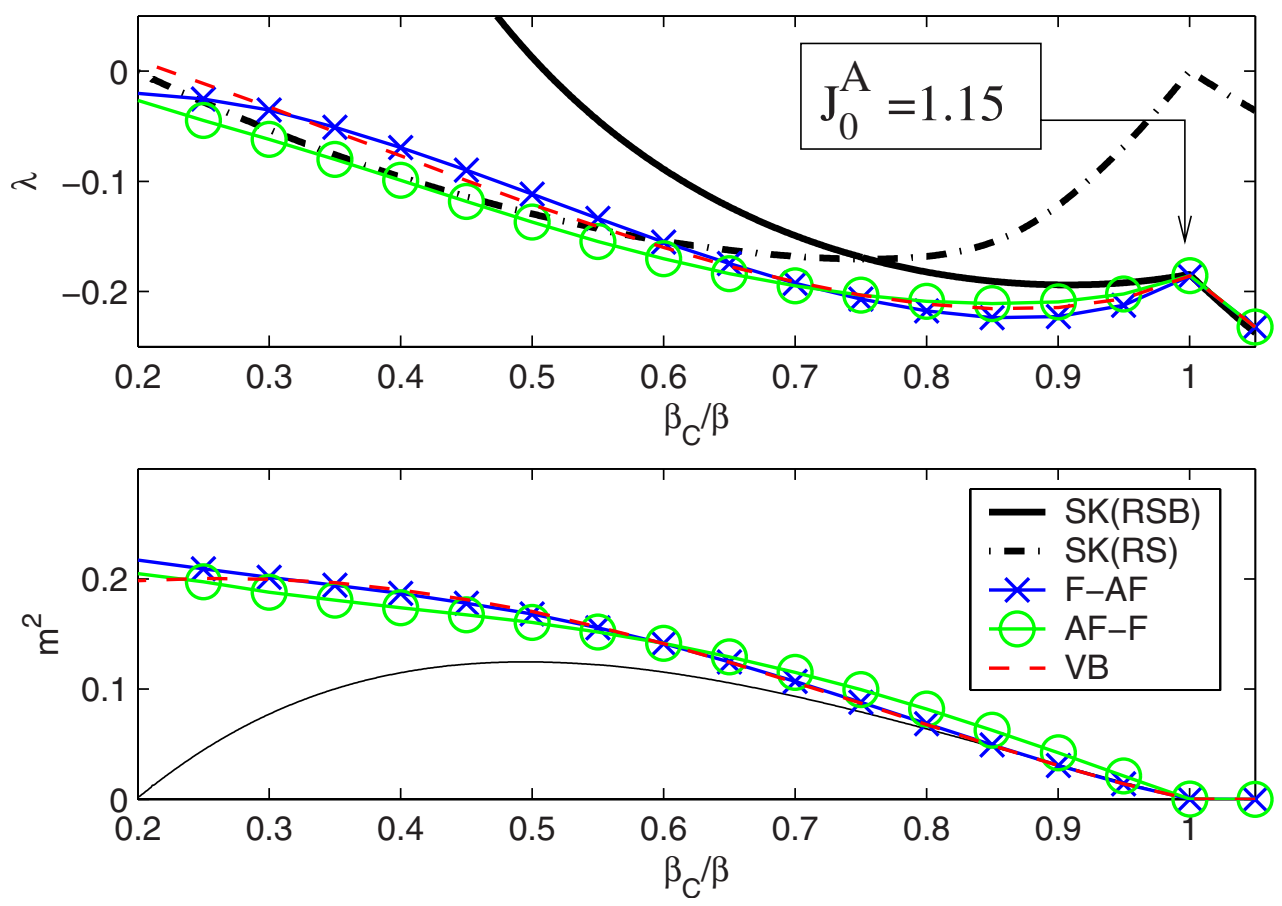

FIG. 10. (Color online) A comparison of the longitudinal stability and magnetization for the F-AF (circles), AF-F (crosses), VB (dashed line), and SK (solid line) models under the RS assumption. Every model is equivalent at the high-temperature ferromagnetic transition point to an SK model with $J_{0}^{A}=1.15$, and temperature variation is considered on the rescaled interval $\beta_{C} / \beta=[0.2,1.05]$. Two stability exponents are given for SK. The marginal stability at the paramagnetic-ferromagnetic transition point $\left(\beta_{C} / \beta=1\right)$ is with respect to a linear instability, which is captured by the longitudinal instability exponent $[\mathrm{SK}(\mathrm{RS})]$, but not by the other nonlinear stability exponents. Properties of the F-AF model display features of the VB model rather than the auxiliary model predictions (Fig. 5). Trends also differ in AF-F model from Fig. 6 , as instability is not realized until much lower than the predicted temperature, properties are again closer to the VB model.

8-10 follow the dashed lines in Figs. 5 and 6 to highlight the behavior of the system as temperature is lowered along fixed $J_{0}^{A}$ values. Figure 8 demonstrates the results for the set of systems equivalent at the high-temperature transition point to a dense model with $J_{0}^{A}=0.925$. In all systems, there is a hightemperature paramagnet to spin glass transition at $\beta_{C}=1$, behavior is examined for relative temperature $\beta_{C} / \beta$ in the interval $(0.1,1.05)$.

The stability exponent $(\lambda)$ and magnetization $\left(\mathrm{m}^{2}\right)$ are identical in all the models very close to the transition, the phase is a spin glass $(m=0, q>0)$, and the RS description is unstable $(\lambda>0)$. The F-AF model becomes unstable toward a mixed (unstable RS ferromagnetic) phase at relatively high temperature. This is qualitatively similar to the prediction based on the auxiliary model of the composite system (see Fig. 5], and the transition temperature is comparable to what would be predicted by the auxiliary model.

When the magnetization is zero (the spin-glass solution), only the even moments of the distribution in the composite models contribute to their behavior. These include only sparse model-dependent parts for F-AF, AF-F so that these models are described by a saddle-point solution identical to the sparse model.

In the AF-F model, the ferromagnetic order parameter is suppressed down to a temperature $\beta_{C} / \beta \approx 0.25$, where it acquires a small value. This is close to the point where $q$ reaches a maximum value, saturation is reached before $q$ $=1$ due to the disconnected component in the sparse substructure. This low-temperature transition must have a strong dependence on higher-order moments since it is in strong contrast with the auxiliary model prediction (Fig. 6).

Figure 9 demonstrates results for the same models and temperature range, but for cases in which the models have a high-temperature triple-point transition. In this figure, the F-AF model has a behavior that is clearly distinct from the other three models. As temperature is lowered, a ferromagnet phase is found rather than a spin-glass phase in the other cases, in agreement with Fig. 5. At lower temperatures, a maximum magnetization is reached and a small decrease in magnetization is discernable at the lowest values in the temperature range. With $\beta_{C} / \beta<0.5$, the RS ferromagnetic phase becomes unstable to a mixed phase.

Initially, at high temperatures, the AF-F model is described by a spin-glass phase. With the continuous emergence of a ferromagnetic moment at low temperature, there is a decrease in the stability exponent.

In Fig. 10, the behavior of systems exhibiting a hightemperature ferromagnetic transition is shown, systems with auxiliary models defined by $J_{0}^{A}=1.15$ at the high-temperature transition. In this regime, re-entrant behavior is seen in the SK model, but not in the VB or composite models. The two composite models follow very closely the behavior of the VB model, although at $\beta_{C} / \beta \sim 0.3$ there appears to be a modification of the trend in the stability exponent for the AF-F model absent in the F-AF and VB models.

The ferromagnetic moment is largest in the AF-F model at high temperature and the F-AF model at low temperatures. There are also several such crossovers in the stability expo- 

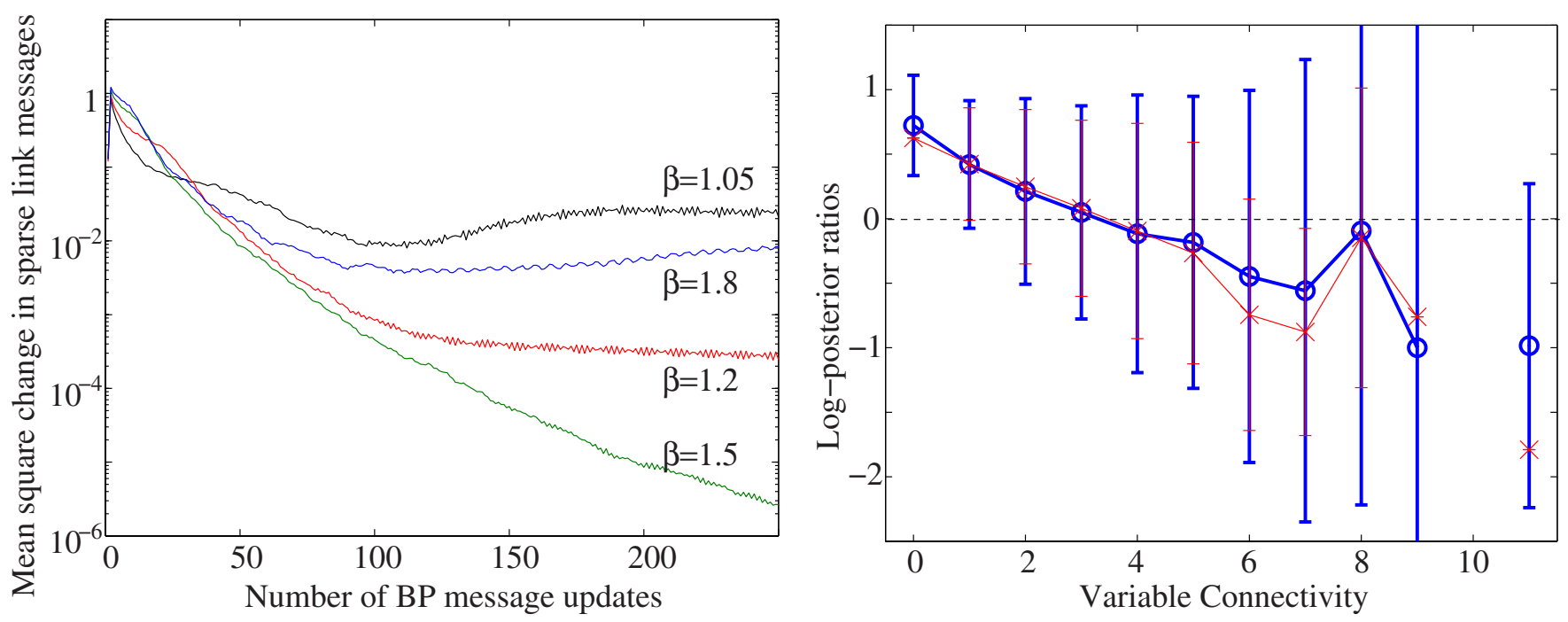

FIG. 11. (Color online) Results in applying BP and Monte Carlo simulation to an F-AF model of size 5000 spins and $\gamma=1$ for various temperatures. Left figure: iteration of BP on a sample graph from various initial conditions is convergent for this sample of quenched disorder at intermediate temperature only, as indicated by the exponential decay in the stability measure. Right: for the case where BP converges $\beta=1.5$, the mean and variance in the field distribution are demonstrated as a function of variable connectivity in the sparse substructure. Thick lines (circles) demonstrate the results of Metropolis-Hastings Monte Carlo simulation. Thin lines (crosses) demonstrate the estimates of BP. These are in agreement except at a high variable connectivity. The magnetism of the system is supported by the alignment of low connectivity variables, with variables of high connectivity in the sparse substructure being magnetized in the opposite direction.

nent. The RS solutions are stable for the composite systems and VB over the full temperature range presented.

\section{RE-ENTRANT BEHAVIOR AND STRUCTURE IN FINITE SYSTEMS}

\section{A. BP and Monte Carlo simulation}

Some testing of thermodynamic results was undertaken in samples of $N=O(100)-O(8000)$ spins by sampling through a Metropolis-Hastings algorithm [24] and estimating logposterior ratios by BP. These studies verified qualitatively the outcomes of the thermodynamic analysis at high temperature. The paramagnetic phase was observed to transform continuously into either a ferromagnetic, spin glass, or mixed (unstable ferromagnetic) phase as temperature was decreased. The ferromagnetic state is assumed to be described by a connected phase space up to finite-size effects. Stability of the BP algorithm was measured through the mean-square change in BP log-posterior estimates [Eq. (30)]

$$
\lambda^{(t)}=\frac{1}{N} \sum_{i=1}^{N}\left(H_{i}^{(t)}-H_{i}^{(t+1)}\right)^{2}
$$

this being a new definition of $\lambda$ related to Eq. (56), but distinguished by the algorithmic context.

Figure 11 demonstrates a simulation of an F-AF model with 5000 spins. This demonstrates that the nonmonotonic behavior seen in the RS solution of the F-AF model and predicted by the leading-order expansion can be realized in finite systems also. The second part of the figure demonstrates the structure of the magnetic phase in the F-AF model. The macroscopic magnetization is supported prima- rily by spins coincident with the disconnected component in the sparse substructure.

\section{B. Structure of phases and transitions}

In the F-AF model, the inhomogeneity in magnetizations, with the disconnected component being the most strongly aligned set of variables, seems an intuitive and necessary feature in a model with such a stark contrast in coupling types.

The disconnected component appears to play an even more vital role in the AF-F model. In the magnetic phase of this model, all the disconnected components are observed in Monte Carlo and BP experiments to be anticorrelated with the macroscopic magnetization, which is an intuitive result. Whereas almost all other variables connected through the sparse substructure take values aligned with the macroscopic order. In the large system limit, there should be some discrimination in the topology within the sparse substructure. Some important topological features of sparse Poissonian graphs are outlined in Fig. 1. In general, the highly connected spins may take one alignment, the disconnected component an opposite alignment, with other variables intermediate.

The inhomogeneity in the structure must also be vital in allowing continuous transitions between various phases and in the dynamics of models. The continuous emergence of a magnetic phase as temperature is lowered in the AF-F model is presumably by a nucleation process, whereas in the F-AF model the ferromagnetic part can emerge first in the disconnected component and percolate inward to the core of the sparse substructure. The absence of sufficient inhomogeneity 
in the regular connectivity models is responsible for the metastability found in some parameter ranges.

\section{SUMMARY}

We have investigated composite models that combine well-studied disordered spin systems with densely and sparsely interacting components with the expectation that the combined model would exhibit features that have not been observed in the original systems. Here we focused on the case of competing interactions of ferromagnetic and antiferromagnetic natures and have explored the phase diagrams for different interaction types and relative strengths. The equilibrium properties were examined through the replica method, exhibiting a new re-entrant behavior from spin glass to ferromagnetic phases as temperature is lowered, and transitions from replica symmetry broken to replica symmetric phases. A discontinuous transition from the paramagnetic to ferromagnetic phases has been observed in regular connectivity graphs.

While the models investigated here comprise classical and well-understood models, they provide insight and understanding of simple complex systems that combine different structures and different levels and interaction types. Such systems have been increasingly the subject of interdisciplinary research activities using methods of varying mathematical rigor. We believe that established methods of statistical physics are highly suitable for understanding both macroscopic and microscopic properties of such systems and that the current investigation paves the way for the study of similar complex systems.

\section{ACKNOWLEDGMENTS}

J.R. is appreciative of the advice given in improving this paper by Juan Neirotti and Ton Coolen. Support from EPSRC Grant No. EP/E049516/1 is gratefully acknowledged.

\section{APPENDIX A: REPLICA CALCULATION}

Carrying out the calculation via the replica method involves a combination of sparse and dense quenched disorder averages. It is convenient to define the sparse substructure in terms of an adjacency matrix A: labeling each edge by $\mu$ and each variable $k, A_{\mu k}=\{0,1\}$. With mean connectivity $C$, the number of edges is $C N / 2$ so that in the absence of other constraints, the probability distribution is defined as

$$
P(\mathrm{~A})=\prod_{\mu=1}^{C N / 2}\left[\left(\begin{array}{l}
N \\
2
\end{array}\right)^{-1} \delta\left(\sum_{k} A_{\mu k}-2\right)\right] .
$$

This is a microcanonical description of interactions, but formulations with the number of edges not strictly fixed (to $C N / 2$ ) are possible. In the limit of large $N$, this describes a Poissonian distribution in the variable connectivity. Both Poissonian and regular connectivity are sufficiently described in typical case analysis by

$$
\begin{aligned}
P(\mathrm{~A}) \propto & \prod_{\mu=1}^{C N / 2}\left[\frac{1}{2} \delta\left(\sum_{k} A_{\mu k}-2\right)\right] \\
& \times \prod_{i=1}^{N}\left\langle\frac{c_{f} !}{C^{c_{f}}} \delta\left(\sum_{\mu} A_{\mu k}-c_{f}\right)\right\rangle \prod_{c_{f} \mu, k} P\left(A_{\mu k}\right),
\end{aligned}
$$

the average in $c_{f}$ being with respect to the marginal variable connectivity distribution of mean $C[25]$ and taking $P\left(A_{\mu k}\right)$ to be a sparse prior

$$
P\left(A_{\mu k}\right)=\left(1-\frac{2}{N}\right) \delta_{A_{\mu k}}+\frac{2}{N} \delta_{A_{\mu k}, 1} .
$$

The Hamiltonian may be written in a form

$$
\mathcal{H}=\frac{1}{2} \sum_{\mu} J_{\mu}^{S}\left[\left(\sum_{k} A_{\mu k} \tau_{k}\right)^{2}-2\right]+\sum_{\langle i j\rangle} J_{\langle i j\rangle}^{D} S_{i} S_{j}
$$

where the representation of the dense part is unmodified from Eq. (1), $J_{\mu}^{S}$ is the random sparse coupling sampled according to $\phi(x)$ in Eq. (7) but can be replaced by the integration variable $x$ in the self-averaged expressions. The replicated partition function is

$$
\left\langle Z^{n}\right\rangle_{\mathcal{Q}}=\prod_{\alpha}\left[\sum_{\vec{S}^{\alpha}}\right]\left\langle\prod_{\mu}\left\langle\exp \left\{\frac{\beta}{2} x \sum_{\alpha}\left[\left(\sum_{k} A_{\mu k} S_{k}^{\alpha}\right)^{2}-2\right]\right\}\right\rangle_{x}\right\rangle_{\mathbb{A}} \prod_{\langle i j\rangle}\left\langle\exp \left\{\beta J_{\langle i j\rangle}^{D} \sum_{\alpha} S_{i}^{\alpha} S_{j}^{\alpha}\right\}\right\rangle_{J_{\langle i j\rangle}^{D}} .
$$

Since the Hamiltonian is factorized with respect to the sparse and dense quenched variables, these averages may be taken independently.

In the sparse part, it is useful to linearize the squared components with a Hubbard-Stratonovich transform for each factor node and replica-index pair

$$
\langle\cdots\rangle_{\mathrm{A}}=\int \prod_{\mu, \alpha}\left[\mathbf{D} \lambda_{\mu}^{\alpha}\right]\left\langle\prod_{\mu}\left\langle\exp \{-\beta x n\} \prod_{k}\left[\exp \left\{\sqrt{\beta x} \sum_{\alpha} \lambda_{\mu}^{\alpha} S_{k}^{\alpha}\right\}\right]^{A_{\mu k}}\right\rangle_{x}\right\rangle_{\mathrm{A}},
$$


with

$$
\int \mathbf{D} \lambda=\frac{1}{\sqrt{2 \pi}} \int d \lambda \exp \left\{-\frac{\lambda^{2}}{2}\right\} .
$$

The delta functions in the adjacency matrix probability distribution (A2) can be represented by complex contour integrals

$$
\begin{aligned}
& \delta\left(L_{\mu}-\sum_{k} A_{\mu k}\right)=\prod_{\mu} \oint_{\mathbf{D}_{2} Y_{\mu} \prod_{k} Y_{\mu}^{A_{\mu k}} ;} \\
& \delta\left(C_{k}-\sum_{\mu} A_{\mu k}\right)=\prod_{k} \oint \mathbf{D}_{C_{k}} Z_{k} \prod_{\mu} Z_{k}^{A \mu k},
\end{aligned}
$$

using the notation

$$
\oint \mathbf{D}_{x} X=\frac{1}{2 \pi i} \oint \frac{d X}{X^{x}} ; \quad \oint \mathbf{D}_{\vec{X}} \vec{X}=\prod_{z} \oint \mathbf{D}_{x_{z}} X_{z} .
$$

The contours can be taken around the origin in the complex plane, so the identities are assured by Cauchy's residue formula.

Factorizing various terms [Eq. (A6)] becomes

$$
\begin{aligned}
\langle\cdots\rangle_{\mathrm{A}} \propto & \left\langle\oint \mathbf{D}_{\vec{C}} \vec{Z} \prod_{\mu}\langle\exp \{-\beta x n\}\right. \\
& \times \int \mathbf{D} \boldsymbol{\lambda} \oint_{\left.\left.\mathbf{D}_{2} Y_{\mu} \prod_{k}\left[\sum_{A_{\mu k}} P\left(A_{\mu k}\right) G\right]\right\rangle_{x}\right\rangle_{\vec{C}},}
\end{aligned}
$$

where $\mathbf{D} \boldsymbol{\lambda} \equiv \Pi_{\mu, \alpha} \mathbf{D}_{\mu}^{\alpha}, G$ abbreviates a number of terms factorized in $k$. Subject to three integrals and an average on connectivities, the dependence on the adjacency matrix is factorized in $\mu$ and $k$, and the trace over A can be completed

$$
\begin{aligned}
\prod_{k}\left[\sum_{A_{\mu k}} P\left(A_{\mu k}\right) G\right]= & \prod_{k}\left[\left(1-\frac{2}{N}\right)\right. \\
& \left.+\frac{2}{N} Y_{\mu} Z_{k} \exp \left\{\sqrt{\beta x} \sum_{\alpha} \lambda_{\mu}^{\alpha} S_{k}^{\alpha}\right\}\right] .
\end{aligned}
$$

Taking the integral in $Y_{\mu}$ picks out only the residue, the second term in the expansion, so that

$$
\prod_{k}\left[\sum_{A_{\mu k}} P\left(A_{\mu k}\right) G\right] \propto \sum_{\langle i j\rangle} \prod_{l=\{i, j\}}\left[\frac{1}{N} Z_{l} \exp \left\{\sqrt{\beta x} \sum_{\alpha} \lambda_{\mu}^{\alpha} S_{l}^{\alpha}\right\}\right] .
$$

Introducing an identity

$$
1=\sum_{\boldsymbol{\sigma}} \prod_{\alpha} \delta_{\sigma^{\alpha}, \sigma_{k}^{\alpha}}
$$

and extracting the $k$ dependence through the order-parameter definition

$$
1=\int \prod_{\boldsymbol{\sigma}}\left[d \Phi(\boldsymbol{\sigma}) \delta\left(\Phi(\boldsymbol{\sigma})-\frac{1}{N} \sum_{k} Z_{k} \delta_{\boldsymbol{\sigma}, \boldsymbol{\sigma}_{k}}\right)\right],
$$

all quenched variable dependence is factorized except in the order-parameter definition. In principle, the identity applies to the entire complex plane, but relevant part of the order parameter is assumed to be real, the imaginary part takes a value 0 in the final saddle-point formulation by assumption. The results are self-consistent given this assumption, and the necessity of real-valued order parameters can be demonstrated in some special cases (see Appendix B).

Having taken the average in A the Hubbard-Stratonovich transform may be inverted to give up to ensemble-dependent constants

$$
\begin{aligned}
\langle\cdots\rangle_{\mathrm{A}}= & \int_{\boldsymbol{\sigma}} d \Phi(\boldsymbol{\sigma}) \prod_{k}\left\langle\frac{C_{k} !}{C^{C_{k}}} \oint \mathbf{D}_{C_{k}} Z_{k}\right. \\
& \left.\times \prod_{\boldsymbol{\sigma}} \delta\left(\Phi(\boldsymbol{\sigma})-\frac{1}{K} \sum_{k} Z_{k} \delta_{S_{k}, \boldsymbol{\sigma}}\right)\right\rangle_{C_{k}} \\
& \times \prod_{\mu}\left[\sum_{\boldsymbol{\tau}, \boldsymbol{\sigma}} \Phi(\boldsymbol{\tau}) \Phi(\boldsymbol{\sigma})\left\langle\exp \left\{\beta x \sum_{\alpha} \tau^{\alpha} \sigma^{\alpha}\right\}\right\rangle_{x}\right],
\end{aligned}
$$

where the average is with respect to the sparse coupling distribution (7).

The dense part of the Hamiltonian can be expanded to second order allowing averages in the quenched couplings,

$$
\langle\cdots\rangle_{J} D=\prod_{\langle i j\rangle}\left[1+\beta \frac{J_{0}}{N} \sum_{\alpha} S_{i}^{\alpha} S_{j}^{\alpha}+\frac{\beta^{2} J^{2}}{2 N} \sum_{\left\langle\alpha_{1}, \alpha_{2}\right\rangle} S_{i}^{\alpha_{1}} S_{i}^{\alpha_{2}} S_{j}^{\alpha_{1}} S_{j}^{\alpha_{2}}\right] .
$$

Defining the dense order parameters,

$$
q_{\alpha}=\frac{1}{N} \sum_{i} S_{i}^{\alpha} ; \quad q_{\left\langle\alpha_{1}, \alpha_{2}\right\rangle}=\frac{1}{N} \sum_{i} S_{i}^{\alpha_{1}} S_{i}^{\alpha_{2}}
$$

the $k$ dependence is extracted

$$
\begin{aligned}
\langle\cdots\rangle_{J} D= & \prod_{\alpha} \delta\left(q_{\alpha}-\frac{1}{N} \sum_{k} S_{k}^{\alpha}\right) \prod_{\left\langle\alpha_{1}, \alpha_{2}\right\rangle} \delta\left(q_{\left\langle\alpha_{1}, \alpha_{2}\right\rangle}\right. \\
& \left.-\frac{1}{N} \sum_{k} S_{k}^{\alpha_{1}} S_{k}^{\alpha_{2}}\right) \exp \left\{\frac{N \beta J_{0}}{2} \sum_{\alpha} q_{\alpha}^{2}\right\} \\
& \times \exp \left\{\frac{N \beta^{2} J^{2}}{2} \sum_{\left\langle\alpha_{1}, \alpha_{2}\right\rangle} q_{\left\langle\alpha_{1}, \alpha_{2}\right\rangle}^{2}\right\} .
\end{aligned}
$$

The definitions of $q_{\alpha}, q_{\left\langle\alpha_{1}, \alpha_{2}\right\rangle}$, and $\Phi$, introduced as $\delta$ functions may be Fourier transformed introducing conjugate parameters, for the real part of $\Phi(\boldsymbol{\sigma})$ 


$$
\begin{aligned}
\delta\left(\Phi(\boldsymbol{\sigma})-\frac{1}{N} Z_{k} \sum_{k} \delta_{\boldsymbol{\sigma}, \boldsymbol{\sigma}_{k}}\right)= & \int_{i \infty}^{-i \infty} \exp \{-C N \hat{\Phi}(\boldsymbol{\sigma}) \hat{\Phi}\} \\
& \times \exp \left\{-C \sum_{k} Z_{k} \hat{\Phi}\left(\boldsymbol{\sigma}_{k}\right)\right\} .
\end{aligned}
$$

By either an explicit calculation of contour integrals, or by a self-consistent assumption, the integral on the complex line (the standard definition of the Fourier transform) might be considered to be rotated onto the real line so that real-valued conjugate parameters can be considered.

The scaling of the Fourier transform by $N$ reflects an assumption of extensive entropy and is also a necessary feature in scalable solutions of the saddle-point method. With the specific choice, the order parameter $\Phi$ is normalized. The choice of an additional factor $C$ in the sparse order-parameter definition is chosen so that $\hat{\Phi}$ and $\Phi$ are normalized.

The trace over replicated spins is finally taken to give an expression for free energy (17), composed of terms (18) and $(20)$, and $\left(\mathcal{G}_{2}\right)$ up to ensemble-dependent constant terms and $O(1 / N)$ corrections. The $\mathcal{G}_{2}$ term is Eq. (19) in the Poissonian variable connectivity, and in general

$$
\begin{aligned}
\mathcal{G}_{2}= & -\log \left\langle\sum _ { S } [ \hat { \Phi } ( S ) ] ^ { c _ { f } } \operatorname { e x p } \left\{\sum_{\alpha} \hat{q}_{\alpha} S_{\alpha}\right.\right. \\
& \left.\left.+\sum_{\left\langle\alpha_{1}, \alpha_{2}\right\rangle} \hat{q}_{\left\langle\alpha_{1}, \alpha_{2}\right\rangle} S^{\alpha_{1}} S^{\alpha_{2}}\right\}\right\rangle_{c_{f}},
\end{aligned}
$$

where the average over $c_{f}$ is with respect to the marginal variable connectivity distribution, uniform, or Poissonian.

\section{Modifications to the saddle-point equations}

The saddle-point equations can be written down for the general case (A20), the generalization of Eq. (21) in the sparse order parameter is

$$
\begin{aligned}
\Phi(\boldsymbol{\sigma}) \propto\left\langlec _ { f } [ \hat { \Phi } ( \boldsymbol { \sigma } ) ] ^ { c _ { f } - 1 } \operatorname { e x p } \left\{\sum_{\alpha} \hat{q}_{\alpha} \sigma^{\alpha}\right.\right. \\
\left.\left.+\sum_{\left\langle\alpha_{1}, \alpha_{2}\right\rangle} \hat{q}_{\left\langle\alpha_{1}, \alpha_{2}\right\rangle} \sigma^{\alpha_{1}} \sigma^{\alpha_{2}}\right\}\right\rangle_{c_{f}} .
\end{aligned}
$$

The dense order parameters are determined through the recursions

$$
q_{\alpha}=\sum_{\boldsymbol{\sigma}} \sigma_{\alpha} \mathcal{P}(\boldsymbol{\sigma}) ; \quad q_{\left\langle\alpha_{1}, \alpha_{2}\right\rangle}=\sum_{\boldsymbol{\sigma}} \sigma^{\alpha_{1}} \sigma^{\alpha_{2}} \mathcal{P}(\boldsymbol{\sigma}),
$$

subject to a normalized distribution

$$
\mathcal{P}(\boldsymbol{S})=\left\langle[\hat{\Phi}(\boldsymbol{S})]^{c_{f}} \exp \left\{\sum_{\alpha} \hat{q}_{\alpha} S_{\alpha}+\sum_{\left\langle\alpha_{1}, \alpha_{2}\right\rangle} \hat{q}_{\left\langle\alpha_{1}, \alpha_{2}\right.} S^{\alpha_{1}} S^{\alpha_{2}}\right\}\right\rangle_{c_{f}} .
$$

The conjugate saddle-point equations are unchanged in form (23).

\section{APPENDIX B: ORDER-PARAMETER CONSIDERATIONS}

An interpretation for some parameters can be gained by consideration of derivatives of the free energy with respect to $\beta$ and simple random external fields $\vec{z}$. This may also be used to prove the consistency of some method assumptions in the case of replica symmetry. The choice of a random field is primarily to allow a concise inclusion within the replica method equations. It is equivalent to working directly with fields that are conjugate to quantities such as $\sum_{\langle i j\rangle} \tau_{i} \tau_{j}$ or with annealed random fields in some cases.

A derivative of $\beta f_{\mathcal{E}}$, with respect to $\beta$ determines the energy density. It is well known that the entropy becomes negative in both the VB and SK models at low temperature if incorrect symmetry assumptions are used; this is also observed in some of the systems presented. Consider also a perturbation on the Hamiltonian to include a nonzero external field

$$
\Delta \mathcal{H}(\vec{\tau})=\sum_{i} z_{i} \tau_{i} ; \quad z_{i}=z \eta_{i}
$$

where $\eta_{i}$ is either uniform or randomly sampled from a symmetric distribution, and $z$ is small and positive. In the case that $\eta_{i}$ is uniform, the derivative of the free-energy density with respect to $z$ in the limit of small $z$ is easy to evaluate and gives the magnetization, which is coincident with the sum over first replica moments at the saddle-point in the selfaveraging case

$$
m=\frac{\partial}{\partial n} \mid \sum_{\alpha} q_{\alpha}^{*}=\frac{1}{N} \sum_{i}\left\langle\tau_{i}\right\rangle .
$$

The derivative when $\vec{\eta}=\{\overrightarrow{1},-\overrightarrow{1}\}$ is coincident with the susceptibility and also the sum over 2 -spin correlations at the saddle-point

$$
\chi_{\text {Lin }}=\frac{\partial}{\partial n} \mid \sum_{n=0\left\langle\alpha_{1}, \alpha_{2}\right\rangle} q_{\left\langle\alpha_{1}, \alpha_{2}\right\rangle}^{*}=\frac{1}{N} \sum_{\langle i j\rangle}\left\langle\tau_{i} \tau_{j}\right\rangle-\left\langle\tau_{i}\right\rangle\left\langle\tau_{j}\right\rangle .
$$

These are useful quantities in evaluating the emergence of ferromagnetic order and in determining phase transitions.

\section{Assumption of real-valued integration variables}

An assumption of the saddle-point method used to evaluate the exponential term describing the free energy is that only real-valued integration parameters (order parameters) need be considered. This appendix demonstrates that any physical solution must be real valued in its first two moments; this is assumed to extend to higher-order moments in the sparse order parameter $\Phi$.

A useful variation in Eq. (B1) for purposes of general analysis are identified by a class of fields aligned with interactions. Consider the factor graph representation of the Hamiltonian (A4). Each of $O(N)$ sparse interactions also has a unique label $\mu=\langle i j\rangle$, with other ordered edges $\langle i j\rangle$ subject to weak (dense) interactions. Therefore, 


$$
z_{i}=\eta_{i} \sum_{j \backslash i}\left(z^{D} J_{(i, j)}^{D}+z^{S} J_{(i, j)}^{S}\right)+\sum_{j \backslash i} \zeta_{(i, j)}\left(z^{D} J_{(i, k)}^{D}+z^{S} J_{(i, j)}^{S}\right)
$$

is well defined and includes a component dependent on the sparse substructure and one on the dense substructure. Each of $\eta_{i}, \zeta_{(i, j)}$ are assumed to be 0 (a default for discussions), uniform (1), or quenched variables sampled independently from $\{-1,1\}$, with $\left\{z^{S}, z^{D}\right\}$ being infinitesimal real positive fields. Unordered matrices are used in Eq. (B4) to describe their ordered counterparts, so that $(i, j)$ is $\langle i j\rangle$ or $\langle j i\rangle$ as ordering dictates, for each ordered pair only one quenched parameter exists.

Physical interpretation for these fields is as follows whenever $J_{0} \neq 0$ and $\eta_{k}=1$ derivatives with respect to $z^{D}$ give a magnetization. When $\eta_{k}=\{-1,1\}$ susceptibilities such as Eq. (B3) are determined. When $\zeta_{(i, j)}=1$, the derivative probes alignments of variables with couplings, again giving a measure that can distinguish an ordered phase from a paramagnetic one. The more complicated physical quantities involve a quenched random field $\zeta_{\langle i j\rangle}=\{-1,1\}$, for example,

$$
\begin{aligned}
\left.\frac{\partial}{\partial\left[\beta\left(z^{D}\right)^{2}\right]}\right|_{z^{D=0}} f_{\mathcal{E}}= & \lim _{N \rightarrow \infty} \frac{1}{2 N}\left\langle\left(\sum_{(i, j)} J_{(i, j)}^{D} S_{i}\right)^{2}\right\rangle \\
& -\frac{1}{2 N}\left\langle\left(\sum_{(i, j)} J_{(i, j)}^{D} S_{i}\right)\right\rangle^{2},
\end{aligned}
$$

identifying a type of susceptibility.

The free energy in the replica formulation, with inclusion of these infinitesimal fields involves a modification of the factor-centric $\left(\mathcal{G}_{1}\right)$ term (18) in the free energy. Following Appendix A,

$$
\begin{aligned}
\mathcal{G}_{1}= & -\sum_{\alpha} \frac{1}{2} \beta J_{0}\left(q_{\alpha}+2 z^{D}\left\langle\zeta_{\langle i j\rangle}\right\rangle\right) q_{\alpha} \\
& -\frac{1}{2} \sum_{\left\langle\alpha_{1}, \alpha_{2}\right\rangle} \beta^{2} J^{2}\left[q_{\left\langle\alpha_{1}, \alpha_{2}\right\rangle}+2\left(z^{D}\right)^{2}\left\langle\left(\zeta_{\langle i j\rangle}\right)^{2}\right\rangle\right] q_{\left\langle\alpha_{1}, \alpha_{2}\right\rangle} \\
& -\frac{C}{2} \log \sum_{S, S^{\prime}} \Phi(S) \Phi\left(S^{\prime}\right) \int d x \phi(x) \\
& \times\left\langle\exp \left\{-\beta x \sum_{\alpha}\left(S^{\alpha}+z^{S} \zeta_{\langle i j\rangle}\right) S^{\prime \alpha}\right\}\right\rangle_{\zeta_{\langle i j\rangle} .} .
\end{aligned}
$$

The variable-centric term is also modified from Eq. (A20), following from Appendix A the averages over $J_{\langle i j\rangle}^{D}$ are straightforward, but the average over $J_{\langle i j\rangle}^{S}$ is involved for the general case, requiring additional order-parameter definitions. Consider the case that $J_{(i, j)}^{S}=J^{S}$ is uniform for simplicity. The expression then becomes

$$
\begin{aligned}
\mathcal{G}_{2}= & -\log \left\langle\sum_{S}[\hat{\Phi}(S)]^{c_{f}} \exp \left\{\sum_{\alpha} \hat{q}_{\alpha} S_{\alpha}+\sum_{\left\langle\alpha_{1}, \alpha_{2}\right\rangle} \hat{q}_{\left\langle\alpha_{1}, \alpha_{2}\right\rangle} S^{\alpha_{1}} S^{\alpha_{2}}\right\}\right. \\
& \left.\times \exp \left\{\beta \eta_{k}\left[c_{f} z^{S}+\left(J_{0}+\lambda J\right) z^{D}\right] \sum_{\alpha} S_{\alpha}\right\}\right\rangle_{c_{f}, \eta_{k}} .
\end{aligned}
$$

with $\lambda$ a normally distributed parameter.
Quantities derived through the proposed choices for $\{\vec{\zeta}, \vec{\eta}\}$ are necessarily real valued at a saddle point and may be calculated in the replica framework given the self-averaged free energy. The derivatives with respect to $\zeta_{\mu}$ are particularly transparent, for example, taking $\eta_{\langle i j\rangle}$ to be a quenched parameter from \pm 1 , the derivative at the saddle-point gives

$$
\left.\frac{\partial}{\partial\left[\beta\left(z^{D}\right)^{2}\right]}\right|_{z^{D}=0} f_{\mathcal{E}}=\left.\frac{\partial}{\partial n}\right|_{n=0} J^{2} \sum_{\left\langle\alpha_{1}, \alpha_{2}\right\rangle} q_{\left\langle\alpha_{1}, \alpha_{2}\right\rangle}^{*},
$$

so that whenever $J^{2}$ is nonzero the RS term, $\Sigma_{\left\langle\alpha_{1}, \alpha_{2}\right\rangle} q_{\left\langle\alpha_{1}, \alpha_{2}\right\rangle}^{*}$ must be real valued. In the case $J^{2}=0$, the order-parameter definition is in any case redundant and can be removed from the free energy. A derivative with respect to $z^{S}$ can produce a similar constraint on the second moment of the sparse distribution [25]. Taking $\eta_{i}$ to be uniform demonstrates that first moments of the order parameter must also be real.

\section{Spin-glass susceptibilities}

The quantity probed through the BP stability analysis is a form of spin-glass susceptibility. Consider two sets of random variables described by a joint probability distribution determined by identical quenched disorder except in a weakfield term

$$
P(\vec{\sigma}, \vec{\tau})=\frac{1}{Z^{2}} \exp \left\{-\mathcal{H}(\vec{\sigma})-\mathcal{H}(\vec{\tau})+z \sum_{k} \eta_{k}\left(z_{1} \sigma_{i}+z_{2} \sigma_{j}\right)\right\},
$$

where $z_{1}, z_{2}$, and $\eta_{k}$ are quenched parameters sampled independently from $\{-1,1\}$, and $z$ is a small external field. Assuming self-averaging, the limit $z \rightarrow 0$ ought to smoothly recover the equilibrium description of a single system (as evaluated using an RS assumption, for example). An expansion of the self-averaged free energy finds at $O\left(z^{2}\right)$ and $O\left(z^{4}\right)$ constant terms and terms dependent on the macroscopic magnetization and susceptibility. These terms are expected to be well defined in the small $z$ limit if the population dynamics method converges, since they are coincident with the RS order-parameter moments at the saddle point, and stability is tested by fluctuations implicit in population dynamics. However, at $O\left(z^{4}\right)$ there is also a dependence on spin-glass susceptibility

$$
\chi_{S G}=\frac{1}{N} \sum_{\langle i j\rangle}\left(\left\langle\sigma_{i} \sigma_{j}\right\rangle-\left\langle\sigma_{i}\right\rangle\left\langle\sigma_{j}\right\rangle\right)^{2} .
$$

The spin-glass susceptibility, as opposed to linear susceptibility (B3), probes a symmetric local instability, symmetries which might allow the linear susceptibility to be convergent are absent. In the replica formulation, nonconvergence of the spin-glass susceptibility in the limit of small $z$ provides sufficient criteria for failure of the RS assumption.

Nonconvergence can be tested by a local stability analysis of the order parameters in a single (uncoupled) model under iteration of the saddle-point equations. The form of spinglass susceptibility tested in the BP framework is not exactly (B10); one must consider an external field reweighted by interaction strengths to determine an appropriately re- 
weighed set of perturbations [Eq. (B4)]. The site factorization is then only achieved by the definition of new order parameters, and it is the linear stability of the original order parameters toward this new description at $z=0$ that is a sufficient test of divergence and comparable to stability of $\mathrm{BP}$ fields under iteration. However, asymptotic divergence of the $\mathrm{BP}$ equations as presented is expected to be sufficient criteria for divergence of the standard spin-glass susceptibility in the equilibrium analysis, accurate as a predictor of trends and parameter dependence.
[1] K. Fischer and J. Hertz, Spin Glasses (Cambridge University Press, Cambridge, England, 1991).

[2] M. Mézard, G. Parisi, and M. Virasoro, Spin Glass Theory and Beyond (World Scientific, Singapore, 1987).

[3] J. Hertz, A. Krogh, and R. Palmer, Introduction to the Theory of Neural Computation (Addison-Wesley, Boston, MA, 1991).

[4] T. Richardson and R. Urbanke, Modern Coding Theory (Cambridge University Press, Cambridge, England, 2008).

[5] H. Nishimori, Statistical Physics of Spin Glasses and Information Processing (Oxford Science, Oxford, London, 2001).

[6] B. Bollobas, Random Graphs, 2nd ed. (Cambridge University Press, Cambridge, England, 2001).

[7] D. Sherrington and S. Kirkpatrick, Phys. Rev. Lett. 35, 1792 (1975).

[8] R. Ellis, Entropy, Large Deviations and Statistical Mechanics (Springer, New York, 1985).

[9] L. Viana and A. Bray, J. Phys. C 18, 3037 (1985).

[10] P. Mottishaw, Europhys. Lett. 4, 333 (1987).

[11] N. S. Skantzos and A. C. C. Coolen, J. Phys. A 33, 5785 (2000).

[12] J. Raymond and D. Saad, J. Stat. Mech.: Theory Exp. 2009,
P05015 (2009)

[13] M. O. Hase and J. F. F. Mendes, J. Phys. A 41, 145002 (2008).

[14] R. Albert, H. Jeong, and A. Barabasi, Nature (London) 406, 378 (2000).

[15] J. Raymond and D. Saad, J. Phys. A 41, 324014 (2008).

[16] R. Monasson, J. Phys. A 31, 513 (1998).

[17] M. Mézard and G. Parisi, Eur. Phys. J. B 20, 217 (2001).

[18] F. Kschischang, B. Frey, and H.-A. Loeliger, IEEE Trans. Inf. Theory 47, 498 (2001).

[19] Y. Kabashima, J. Phys. Soc. Jpn. 72, 1645 (2003).

[20] E. Mallard and D. Saad, Phys. Rev. E 78, 021107 (2008).

[21] O. Rivoire, G. Biroli, O. Martin, and M. Mézard, Eur. Phys. J. B 37, 55 (2004).

[22] J. d. Almeida and D. Thouless, J. Phys. A 11, 983 (1978).

[23] P. Mottishaw and C. De Dominicis, J. Phys. A 20, L375 (1987).

[24] D. Landau and K. Binder, A Guide to Monte Carlo Simulations in Statistical Physics, 2nd ed. (Cambridge University Press, Cambridge, UK, 2005).

[25] J. Raymond, Ph.D. thesis, Aston University, Birmingham, 2008. 